\title{
Dynamics of Protoplanetary Disks
}

\author{
Philip J. Armitage \\ JILA, 440 UCB, University of Colorado, Boulder CO80309-0440, USA
}

\begin{abstract}
Protoplanetary disks are quasi-steady structures whose evolution and dispersal determine the environment for planet formation. I review the theory of protoplanetary disk evolution and its connection to observations. Substantial progress has been made in elucidating the physics of potential angular momentum transport processes including self-gravity, magnetorotational instability, baroclinic instabilities, and magnetic braking - and in developing testable models for disk dispersal via photoevaporation. The relative importance of these processes depends upon the initial mass, size and magnetization of the disk, and subsequently on its opacity, ionization state, and external irradiation. Disk dynamics is therefore coupled to star formation, pre-main-sequence stellar evolution, and dust coagulation during the early stages of planet formation, and may vary dramatically from star to star. The importance of validating theoretical models is emphasized, with the key observations being those that probe disk structure on the scales, between $1 \mathrm{AU}$ and $10 \mathrm{AU}$, where theory is most uncertain.
\end{abstract}

Figures and illustrations from this article are available at http://jila.colorado.edu/ pja/araa.html

\section{CONTENTS}

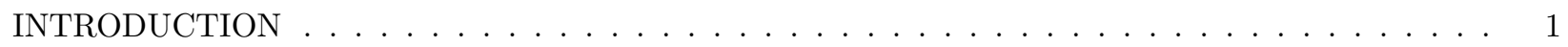

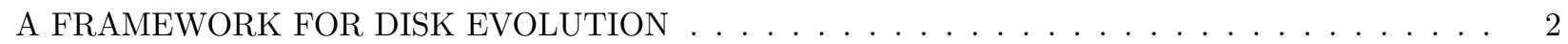

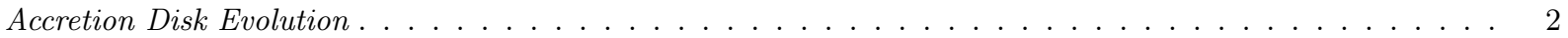

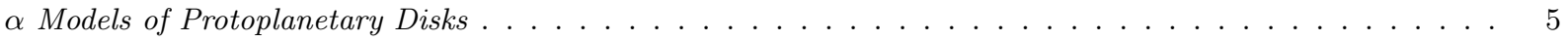

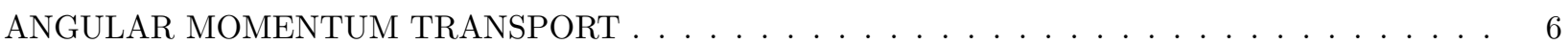

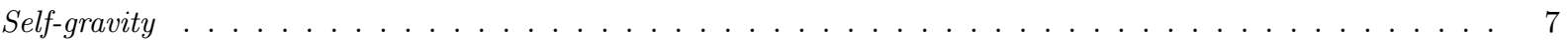

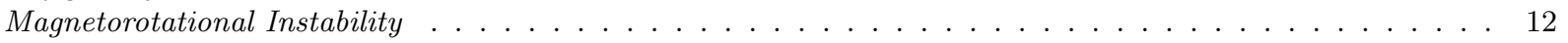

Baroclinic instability and vortices . . . . . . . . . . . . . . . . . . 24

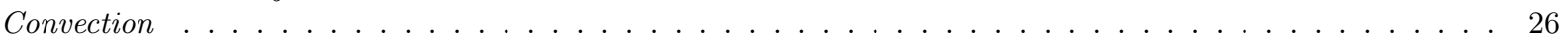

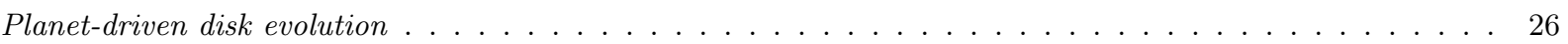

Section summary . . . . . . . . . . . . . . . . . . . . . . . . . . . 27

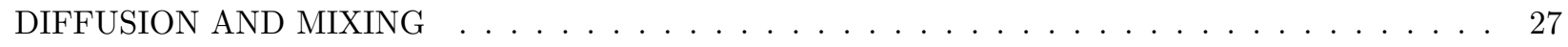

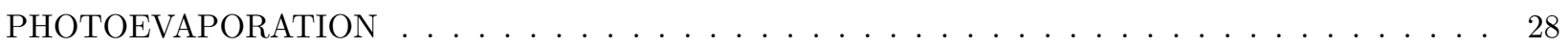

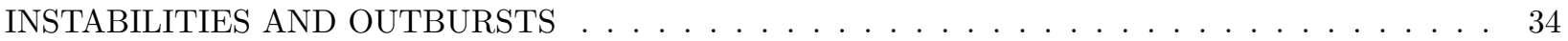

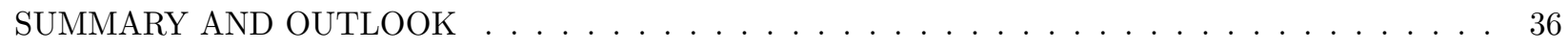

\section{INTRODUCTION}

Protoplanetary disks are the observational manifestation of the initial conditions for planet formation. They can be defined as rotationally supported structures of gas (invariably containing 
dust) that surround young, normally pre-main-sequence stars. Although most observed disks have inferred masses that are a small fraction of the stellar mass, no meaningful distinction can be drawn between physical processes in protoplanetary disks and those that occur in the earlier, "protostellar" phase, in which both star and disk are accreting rapidly. Similarly, a common set of processes operate, albeit to varying degrees, in disks around brown dwarfs, Classical T Tauri stars (low-mass pre-main-sequence stars that are actively accreting), and massive stars. A clear demarcation does separate protoplanetary disks from debris disks; dusty gas-poor structures around older stars whose properties reflect the collisional evolution of a population of small bodies (Wyatt 2008).

Around low-mass stars, protoplanetary disks are persistent; the typical lifetime of $\sim 10^{6}$ years (Haisch, Lada \& Lada 2001) equates to thousands of dynamical times at 100 AU. Evolution can only rarely be observed in individual objects, and must instead be discerned from statistical studies of populations. This slow evolution is a consequence of angular momentum conservation; protoplanetary disks are nearly stable fluid configurations that evolve under the action of relatively slow processes - angular momentum transport, mass infall, and disk winds. These agents control both the secular evolution and the eruptive behavior observed in protoplanetary disks, and set the overall environment within which planet formation occurs: the location of the snow line, the mass and time available to form gas giants, the rate of migration, and so forth. Moreover, in most models the evolution of the disk is linked to the presence of turbulence within the disk gas, and the strength and nature of that turbulence controls the growth of solids during the earliest phases of planet formation (for a review, see Armitage 2010).

A generic model for the evolution of protoplanetary disks, based on the theory of geometrically thin accretion disks (Lynden-Bell \& Pringle 1974), has long been available, but this model lacks predictive power absent a detailed specification of the angular momentum transport and mass loss processes at work. Pinning these down has taken a long time, but substantial progress has now been made in understanding candidate angular momentum transport and mass loss mechanisms. This review addresses the physics of these mechanisms, how they may combine to yield some of the phenomena known to occur in disks around young stars, and how they relate to properties of disks that may be observable in the future. The focus is theoretical, observational aspects of disk structure and evolution are discussed in the review by Williams \& Cieza (2011) in this volume.

\section{A FRAMEWORK FOR DISK EVOLUTION}

Protoplanetary disks are observed to be geometrically thin, in the sense that the vertical scale height $h \ll r$, and typically have inferred masses $M_{\text {disk }} \ll M_{*}$. These properties imply that models of disk evolution should be grounded within the theory of thin accretion disks (Pringle 1981), within which radial pressure gradients are negligible, the angular velocity of the fluid is that of a particle in a point mass potential, $\Omega_{\mathrm{K}}=\sqrt{G M_{*} / r^{3}}$, and the specific angular momentum is an increasing function of radius, $l \propto \sqrt{r}$. Accretion then requires that the accreting gas must lose angular momentum, and the central problem of thin disk theory is to determine why this should occur. For protoplanetary disks, there are two qualitatively distinct possibilities; angular momentum may be redistributed within the disk ("viscous" disk models, which for reasons of space are the exclusive focus of this review) or it may be lost to an external sink ("magnetic wind" or "magnetic braking" models).

\subsection{Accretion Disk Evolution}

For a thin disk, the hydrostatic vertical structure largely decouples from the equations describing the radial evolution, which can be simplified by integrating over the vertical thickness (a clear textbook treatment is given by Frank, King \& Raine 2002). In this limit, the surface density $\Sigma(r, t)$ 
of an axisymmetric planar disk in a Keplerian potential satisfies,

$$
\frac{\partial \Sigma}{\partial t}=\frac{3}{r} \frac{\partial}{\partial r}\left[r^{1 / 2} \frac{\partial}{\partial r}\left(\nu \Sigma r^{1 / 2}\right)\right]
$$

provided that external torques and mass loss can be neglected. This equation follows from the conservation of mass and angular momentum, and is exactly true for a viscous fluid, where $\nu$ would be the kinematic viscosity. Under normal conditions (unless $\partial(\nu \Sigma) / \partial \Sigma<0$ ) solutions to the equation show that gas in the disk will accrete onto the star while simultaneously spreading diffusively to large radii. Less obviously, it is also valid if $\nu$ is an effective viscosity arising from turbulence within the disk, provided that the turbulence can be described as a local process (Balbus \& Papaloizou 1999). Likewise, either a microscopic viscosity or local turbulence results in a rate of energy dissipation per unit surface area of the disk,

$$
Q_{+}=\frac{9}{8} \nu \Sigma \Omega_{\mathrm{K}}^{2}
$$

where $\Omega_{\mathrm{K}}$ is the Keplerian angular velocity. At radii where the disk can be considered to be in a steady-state, these relations immediately specify the variation of $\Sigma$ and effective temperature $T_{\text {eff }}$ with radius (ignoring for now stellar irradiation),

$$
\begin{aligned}
\nu \Sigma & =\frac{\dot{M}}{3 \pi}\left[1-\sqrt{\frac{r_{\text {in }}}{r}}\right] \\
T_{\mathrm{eff}}^{4} & =\frac{3 G M_{*} \dot{M}}{8 \pi \sigma r^{3}}\left[1-\sqrt{\frac{r_{\text {in }}}{r}}\right] .
\end{aligned}
$$

Here, $\dot{M}$ is the accretion rate, $\sigma$ the Stefan-Boltzmann constant, and we have assumed vanishing torque at an inner radius $r_{\text {in }}$.

Up to this point, we have gotten away with only very limited assumptions (that the turbulence is local, and that there are no external torques), but in return we have only predicted one quantity, the effective temperature profile of a steady disk. To proceed further, we must specify $\nu$. Here, there are two possibilities. The classical approach, which still informs the language and much of the work oriented toward the observational modeling of disks, is to postulate a simple scaling relation between $\nu$ and some locally defined property of the flow. Almost invariably, one takes (Shakura \& Sunyaev 1973),

$$
\nu=\alpha \frac{c_{s}^{2}}{\Omega_{\mathrm{K}}}=\alpha c_{s} h,
$$

where $c_{s}$ is the disk sound speed and the second equality follows from vertical hydrostatic equilibrium $\left(h \simeq c_{s} / \Omega_{\mathrm{K}}\right)$. The parameter $\alpha$ is a dimensionless function that will be a constant if the assumed scaling law is obeyed. A variety of simple arguments can be used to motivate this scaling, but at heart it is a guess whose fidelity must be checked through observations and more detailed calculations. Its appeal lies in its simplicity; if we assume that $\alpha$ is a constant then we can readily construct a model for both the thermal structure (via Equation 2) and evolution (Equation 1 ) of the protoplanetary disk, that has just one free parameter.

A more predictive approach is to try and compute the effective viscosity that arises from some turbulent process directly. (Often, though somewhat confusingly, the results of such calculations are reported in terms of an "effective $\alpha$ ".) In the case of disk self-gravity, this can sometimes be accomplished via an analytic thermal equilibrium argument, to be discussed in $\$ 3.1$. More typically, however, there is no alternative but to compute the non-linear structure of the turbulence and evaluate the resultant stresses. Working in cylindrical polar co-ordinates $(r, \phi, z)$, the dominant 
components of the stress tensor can be written as a density-weighted average of the fluctuating velocity, v, and magnetic fields, B (Balbus \& Hawley 1998),

$$
W_{r \phi}=\left\langle\delta v_{r} \delta v_{\phi}-\frac{B_{r} B_{\phi}}{4 \pi \rho}\right\rangle_{\rho},
$$

where $\delta v_{\phi}=v_{\phi}-r \Omega_{\mathrm{K}}$. Written in this form, we see that the stress is the sum of a fluid component, described as the Reynolds stress, and a magnetic or Maxwell stress. Both components are proportional to the energy density in their respective turbulent fields. The $\alpha$ prescription, in this notation, amounts to writing,

$$
W_{r \phi}=\alpha c_{s}^{2}
$$

from which we see that $\alpha$ will be a constant if the correlated velocity fluctuations, and the Alfvén speeds associated with the appropriate components of magnetic field, both scale with $c_{s}$.

Although there may be a class of astrophysical disk systems in which the constant $\alpha$ approximation works reasonably well, protoplanetary disks are probably not a member. We will elaborate on the reasons for this later, but for now it suffices to note that magnetic fields, which probably play a crucial role in transport (Equation 6), couple to the hot gas in the inner disk quite differently than to the cool or diffuse matter further out. It would be surprising if this did not result in substantial variations in $\alpha$. Observationally, this complexity highlights the need to obtain multiple constraints on the dynamical processes going on within disks, ideally across as wide a range of radii as possible. Many phenomena, some of which are illustrated in Figure 1, offer potential windows into different aspects of the underlying dynamics,

1. Measurements of disk magnetic fields, or of turbulent velocity fields within the disk (Hughes et al. 2010, 2009), yield direct access to the nature of disk turbulence, which is connected to the stress (Equation 6). Dust settling, and radial mixing of gas or particles, also depend upon the turbulence, but these phenomena are less directly related to the stress.

2. Determinations of the surface density profile (e.g. Andrews et al. 2009), or joint measurements of $\Sigma$ and $\dot{M}$, allow the recovery of $\nu(r)$ if the disk is in a steady-state (Equation 3 ).

3. Studies of the evolution of disk populations, for example determination of $\langle\dot{M}(t)\rangle$ from observations of clusters of different ages (e.g. Hartmann et al. 1998), constrain angular momentum transport via the time dependence implied by Equation (1).

Within the basic framework of viscous disk theory, these disparate observables constrain a single underlying driver of evolution: the stress as measured (say) by $\alpha(r, t)$. Demonstrated concordance (or discrepancies) between independent measures of $\alpha$ thus represents an empirical route to checking the basic assumptions of disk theory.

As Balbus \& Papaloizou (1999) have emphasized, the assumption that disks evolve due to local turbulent transport is highly restrictive, because in this regime the stress uniquely determines the energy dissipation rate as well as the angular momentum flux. This need not be true. Angular momentum can be redistributed within disks via laminar magnetic torques or by global waves, and can be lost entirely if the disk is threaded by an open magnetic field that exerts a torque on the disk surface. For an open field with a vertical component $B_{z}$, and an azimuthal component $B_{\phi, s}$ (measured at the disk surface), the ratio of the external torque to internal Maxwell stresses is (e.g. Königl \& Salmeron 2010),

$$
\frac{\mathcal{T}_{\text {ext }}}{\mathcal{T}_{\text {visc }}} \sim \frac{B_{z} B_{\phi, s}}{\left\langle B_{r} B_{\phi}\right\rangle}\left(\frac{h}{r}\right)^{-1} .
$$

Since $(h / r)^{-1} \sim 10$, open magnetic fields can be modestly weaker than turbulent fields within the disk and yet drive significant evolution. The challenge for "pure" disk wind and magnetic braking models (those which assume that internal torques are unnecessary) is to determine whether 


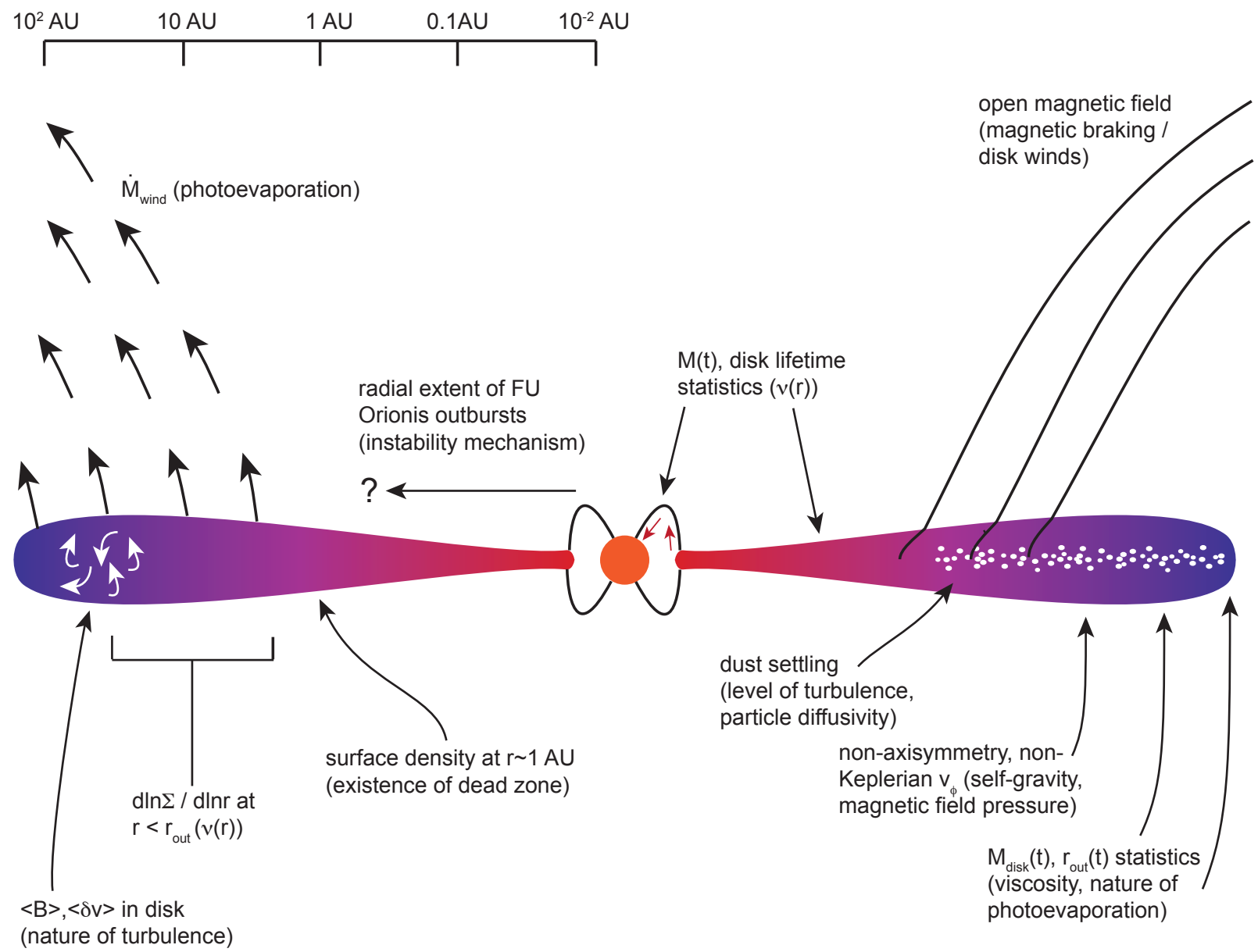

Figure 1: Observable diagnostics of protoplanetary disk dynamics. Measurements of the local strength and coherence of magnetic fields yield constraints on the relative importance of internal versus external angular momentum transport, and on the nature of turbulence. The global surface density profile, at radii where the disk has attained a steady-state, reflects the radial dependence of the efficiency of angular momentum transport. The distribution of quantities such as the disk lifetime, $\dot{M}, M_{\text {disk }}$, and $r_{\text {out }}$, depends primarily upon the average transport efficiency and the rate of wind mass loss. Concordance between multiple independent diagnostics tests the overall paradigm for evolution.

even weak open fields thread disks across a sufficiently wide range of radii, and whether angular momentum loss can occur steadily enough to be consistent with the long lifetime of many observed disks. Of course it is possible to envisage hybrid models in which external torques dominate only in some fraction of cases (perhaps when a collapsing cloud has a particularly strong field), or models in which disk winds are only present at some radii.

\section{$2.2 \alpha$ Models of Protoplanetary Disks}

Absent detailed knowledge of how the efficiency of angular momentum transport scales with the local physical conditions, the simplest approach to constructing viscous disk models is to assume that $\alpha$ is a constant. Computing an " $\alpha$ disk model" of this type requires calculating the vertical 
disk structure as a function of $\Omega_{\mathrm{K}}, \Sigma$ and $\alpha$, taking account of the opacity, the mechanism of energy transport between the mid-plane and the photosphere, and any incident external radiation flux. Detailed $\alpha$ disk models have been constructed by Bell et al. (1997), by D'Alessio et al. (1998), and by others. Models in this class are broadly consistent with both imaging and Spectral Energy Distribution (SED) data for young stars (e.g. D'Alessio, Calvet \& Hartmann 2001), with the main hinderance to a precise test being uncertainties in the evolution of the dust within the disk that furnishes the dominant opacity. The value of $\alpha$ itself can be constrained by studies of the evolution of the stellar accretion rate (Hartmann et al. 1998), or by detailed studies of individual systems (Hueso \& Guillot 2005). These methods typically yield $\alpha \sim 10^{-2}$, with large uncertainties. The constraint on $\alpha$ is comparable to the elementary estimate that can be derived by setting the global evolution time,

$$
t_{\nu} \simeq \frac{r^{2}}{\nu}=\frac{1}{\alpha \Omega_{\mathrm{K}}}\left(\frac{h}{r}\right)^{-2},
$$

equal to $1 \mathrm{Myr}$ at a typical disk scale of $30 \mathrm{AU}$, in a disk with $h / r=0.05$. For planet formation, a significant prediction of $\alpha$ models is that the steady-state surface density profile is roughly $\Sigma \propto r^{-1}$ between a few AU and $\sim 10^{2}$ AU, significantly flatter than the Minimum Mass Solar Nebula profile $\Sigma \propto r^{-3 / 2}$ (Weidenschilling 1977).

Although $\alpha$ models provide a parsimonious description of a substantial body of data in terms of a single free parameter, there are compelling reasons to think that they are not a full description of protoplanetary disks. Put simply, no known angular momentum transport mechanism yields a constant value of $\alpha$ under protoplanetary disk conditions. Transport by magnetohydrodynamic (MHD) processes, for example, is predicted to vary in efficacy with the degree of ionization, which in turn is a function of the temperature and surface density. Similarly, transport by self-gravity, and by possible instabilities that generate vortices, scales with the disk cooling time $\left(t_{\text {cool }}\right.$, the ratio of the disk thermal energy per unit area to the total flux). It is prudent to approach observationally untested $\alpha$ model predictions with caution.

Despite these realities, simple $\alpha$ models continue to represent a useful starting point for disk studies, since they allow a first estimate of how critical quantities such as the ionization rate ought to vary with radius in the disk. In this spirit, Figure 2 shows how several significant radii, relevant to understanding where different angular momentum transport mechanisms may be effective, scale with stellar accretion rate. Although the model used to compute the figure is highly simplified, it exposes several generic features of protoplanetary disks. First, stellar irradiation provides the dominant energy source at large radii, whereas for all but the lowest $\dot{M}$ viscous heating dominates close to the star (D'Alessio et al. 1998). This will be true unless the disk geometry is such as to throw the outer regions into shadow. Second, there is a region at $r \sim 1$ AU where the disk is cool and dense, and thus likely to have a very low ionization fraction. Careful consideration is needed to assess whether MHD processes can provide angular momentum transport in this region. Third, the Toomre $Q$ parameter, which describes the linear stability of a disk to gravitational instability (Toomre 1964), can be low enough for instability to develop at large radii for accretion rates above $\sim 10^{-7} M_{\odot} \mathrm{yr}^{-1}$. Finally, the cooling time scale is initially (when $\dot{M}$ is high) large interior to $r \sim 10^{2}$ AU, but the line of dynamical time scale cooling sweeps inward to small radii as $\dot{M}$ drops and stellar irradiation increasingly dominates the energy budget of the disk.

\section{ANGULAR MOMENTUM TRANSPORT}

The most intensively studied mechanisms for angular momentum transport within viscous protoplanetary disks are self-gravity and the magnetorotational (or Balbus-Hawley) instability. These originate from linear instabilities. Hydrodynamic instabilities associated with the disk's radial thermal structure ("baroclinic instability") could also contribute to angular momentum transport, 


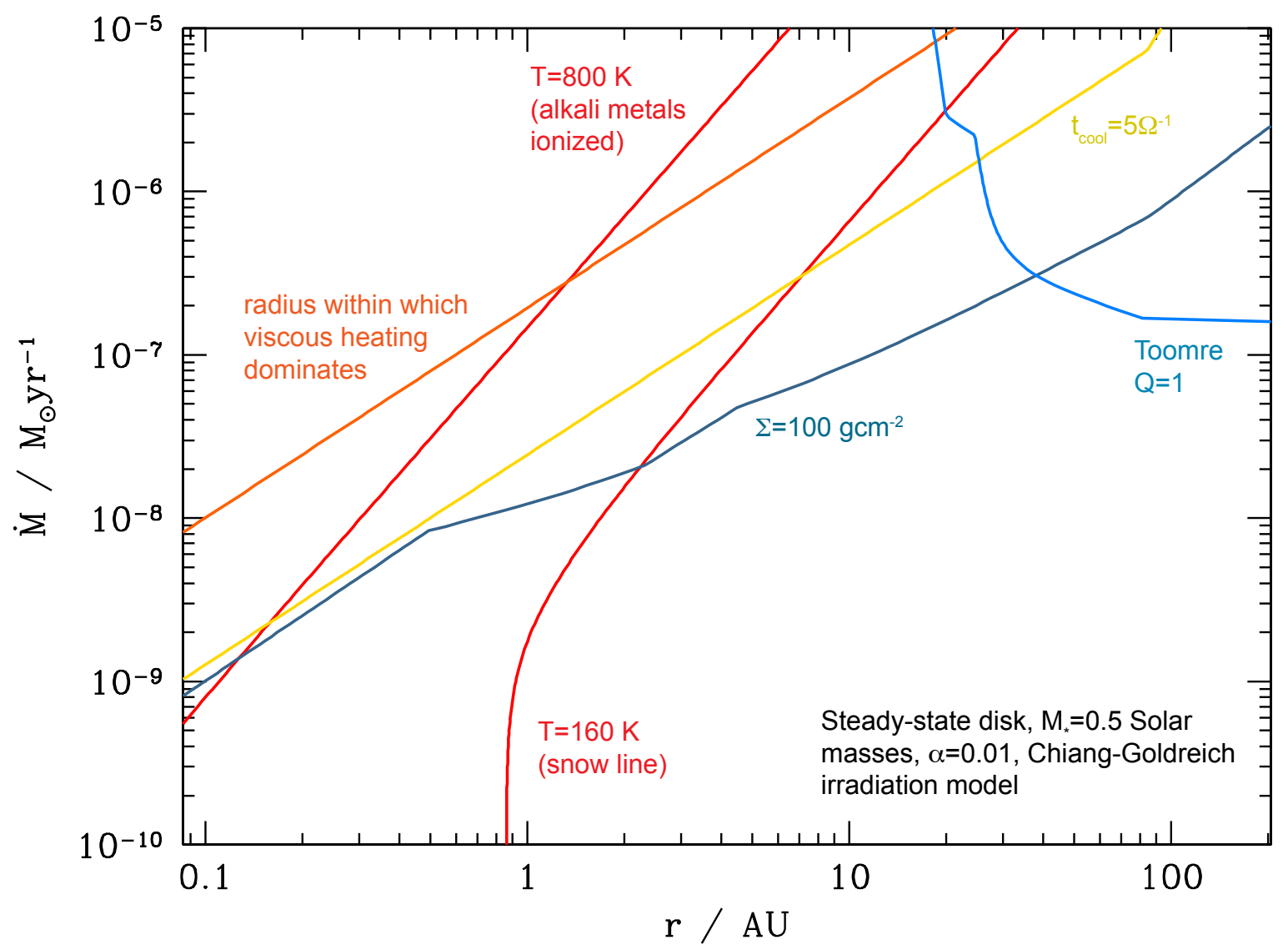

Figure 2: Illustration of how critical disk radii, delimiting different physical regimes relevant to angular momentum transport and planet formation, scale with accretion rate $\dot{M}$. Temperatures are mid-plane values, the cooling time scale is defined as $t_{\text {cool }}=\Sigma c_{s}^{2} / 2 \sigma T_{\text {eff }}^{4}$, and the Toomre $Q$ parameter $Q=c_{s} \Omega_{\mathrm{K}} / \pi G \Sigma$. The plot is based upon a one-zone vertical model (calculated as described, e.g., by Frank, King \& Raine 2002) of a steady-state $\alpha$ disk around a $0.5 M_{\odot}$ star, with $\alpha=10^{-2}$. The opacity includes contributions from water ice, amorphous carbon, silicates and graphite (Z. Zhu, private communication). The calculation is approximate: the radial dependence of stellar irradiation is assumed to follow the Chiang \& Goldreich (1997) form, independent of both time and disk accretion rate.

through the non-linear formation of vortices. In this Section, we review the current understanding these processes. We also discuss, briefly, convection and angular momentum transport catalyzed by embedded planets, which might be important locally in a subset of protoplanetary disks. For want of space the alternate possibility - that angular momentum loss due to magnetic braking is the dominant evolutionary process - is not discussed; the reader seeking details of these models is referred to the comprehensive review by Königl \& Salmeron (2010).

\subsection{Self-gravity}

The fact that the self-gravity of a massive disk, subject to radiative cooling, would result in angular momentum transport and accretion, was recognized by Paczyński (1978). In a non-magnetized disk, the stability of over-dense perturbations is set by the balance between self-gravity, pressure 
(which stabilizes small scales against collapse), and shear (which stabilizes large scales). The linear stability of an initially axisymmetric thin disk, rotating with angular velocity $\Omega(r)$, is quantified by the Toomre $Q$ parameter (Toomre 1964),

$$
Q \equiv \frac{c_{s} \kappa}{\pi G \Sigma}
$$

where $\kappa$, the epicyclic frequency, is the frequency at which a radially displaced particle oscillates. Instability to the growth of axisymmetric perturbations occurs for $Q \lesssim 1$, though more general disturbances (Papaloizou \& Savonije 1991) can grow whenever $Q \lesssim 1.5$. In the absence of an external potential, the epicyclic frequency for a low mass disk $\left(M_{\text {disk }} \ll M_{*}\right)$ can be written as $\kappa^{2} \equiv(2 \Omega / r) \mathrm{d} / \mathrm{d} r\left(r^{2} \Omega\right) \simeq \Omega_{\mathrm{K}}^{2}$. This simplification is often used for protoplanetary disks. If the gas has mean molecular weight $\mu$, attaining $Q=1$ requires that the surface density satisfy,

$$
\Sigma>7.9 \times 10^{2}\left(\frac{\mu}{2.4}\right)^{-1 / 2}\left(\frac{M_{*}}{M_{\odot}}\right)^{1 / 2}\left(\frac{r}{10 \mathrm{AU}}\right)^{-3 / 2}\left(\frac{T_{c}}{20 \mathrm{~K}}\right)^{1 / 2} \mathrm{~g} \mathrm{~cm}^{-2},
$$

where $T_{c}$ is the mid-plane temperature. To express this more intuitively, we note that the mass enclosed within radius $r$ is, approximately, $M_{\text {disk }}(r) \sim \pi r^{2} \Sigma$. Noting that $h=c_{s} / \Omega_{\mathrm{K}}$, an equivalent but approximate local condition for instability is,

$$
\frac{M_{\text {disk }}(r)}{M_{*}} \gtrsim \frac{h}{r}
$$

Gravitational instability is thus likely to occur early, when protoplanetary disks are still massive, and at large radii. Indeed, if irradiation can be ignored (usually, it cannot), a steady-state $\alpha$ disk will always become Toomre unstable beyond some critical radius. Once a disk becomes gravitationally unstable, numerical simulations are required in order to assess the subsequent evolution (Laughlin \& Bodenheimer 1994). Three broad classes of outcomes have been identified. The disk may settle into a quasi-steady, "saturated" state of self-gravitating turbulence, in which trailing spiral arms yield an outward transport of angular momentum via gravitational torques (Lynden-Bell \& Kalnajs 1962). Alternatively, the disk may exhibit large amplitude bursts of accretion, or it may fragment and break up into distinct bound objects. The first two modes are relevant to disk evolution, while the possibility of fragmentation is of great interest as a mechanism for forming massive planets or substellar objects (Boley 2009; Boss 1997; Stamatellos, Hubber \& Whitworth 2007).

Although the local linear stability of a gas disk is only a function of its instantaneous structure the surface density and sound speed - the non-linear evolution depends critically upon the thermodynamic and radiative properties of the disk (Nelson, Benz \& Ruzmaikina 2000; Pickett et al. 1998, 2000). The importance of cooling for the non-linear behavior can be demonstrated, in part, using elementary arguments, provided that we stipulate that self-gravitating disk turbulence acts as a local process (Paczyński 1978). In this limit, the constraint implied by the linear stability threshold (Equation 10 allows us to determine an explicit form for $\alpha\left(\Sigma, \Omega_{\mathrm{K}}\right)$. We first note that for a disk primarily heated by viscous dissipation, rather than irradiation, thermal equilibrium implies that the heating rate per unit area, $(9 / 4) \nu \Sigma \Omega_{\mathrm{K}}^{2}$, must balance cooling at a rate $2 \sigma T_{\mathrm{eff}}^{4}$. In terms of $\alpha$,

$$
\alpha=\frac{4}{9 \gamma(\gamma-1)} \frac{1}{t_{\mathrm{cool}} \Omega_{\mathrm{K}}},
$$

where $\gamma$ is the adiabatic index, $t_{\text {cool }}=U / 2 \sigma T_{\text {eff }}^{4}$ and $U$, the thermal energy per unit surface area, is given by $U=c_{s}^{2} \Sigma / \gamma(\gamma-1)$. This form for $\alpha\left(t_{\text {cool }}\right)$ is an identity for viscous disks (see e.g. Pringle 1981, §7), but it does not generally determine $\alpha$ because $T_{\text {eff }}(\Sigma)$, which is required to determine $t_{\mathrm{cool}}$, is unknown. For a disk in which self-gravity is the sole source of angular momentum transport, however, we have the advantage of an additional constraint; $\Sigma$ must be related to the central sound 
speed via Equation (10). If we take $Q_{0}$, the value of the Toomre parameter in the saturated state of gravitational instability, to be a constant (thereby anticipating the result that a self-gravitating disk tends to maintain a state of marginal instability with $Q_{0}>1$ ), then $c_{s}=\pi G Q_{0} \Sigma / \Omega_{\mathrm{K}}$. Making this assumption, we use the one-zone approximation to the equation of radiative diffusion in $z$ to relate the central temperature, $T_{c}$, to the effective temperature $T_{\text {eff }}$. For an optically thick disk (for details, see e.g. Hubeny 1990),

$$
T_{c}^{4} \sim \tau T_{\mathrm{eff}}^{4},
$$

where $\tau$, the optical depth between the disk surface and mid-plane, is given in terms of the midplane opacity by $\tau=(1 / 2) \Sigma \kappa$. Writing $\kappa=\kappa_{0} T_{c}^{\beta}$, one finds that $\alpha$ can be expressed as an explicit function of just the surface density and angular velocity (Levin 2007),

$$
\alpha \sim \frac{\sigma}{\kappa_{0}}\left(\frac{\mu m_{p}}{\gamma k_{B}}\right)^{4-\beta}\left(Q_{0} \pi G\right)^{6-2 \beta} \Sigma^{4-2 \beta} \Omega^{2 \beta-7} .
$$

Here we have omitted numerical prefactors, the exact values of which vary in the literature depending upon the assumed vertical structure (which has not been accurately determined from simulations), and with whether the sound speed used is isothermal or adiabatic. Analogous expressions can be derived for optically thin disks, or for disks with more general opacity laws that are a function of density as well as temperature (Cossins, Lodato \& Clarke 2010).

The simplicity of Equation 15 results from the assumption that angular momentum transport due to self-gravity can be treated as a local, "gravitoturbulent", process. Since gravity is a long-range force, the general validity of the local approximation is questionable, and it is easy to envisage circumstances in which it would fail entirely. A disk that develops a strong bar-like structure, for example, could have regions in which gravitational torques from the bar remove angular momentum and drive accretion without the attendant energy dissipation that occurs in a local model. More formally, Balbus \& Papaloizou (1999) show that a self-gravitating disk can only be described by a local $\alpha$ model if, at all radii, the pattern speed of the waves $\Omega_{p}$ matches the local angular velocity $\Omega$. If, on the other hand, $\Omega_{p}$ is very different from $\Omega$, the radial energy flux associated with waves in the disk will violate the assumption of locality and mandate a global treatment of the disk dynamics.

The locality of angular momentum transport by disk self-gravity has been tested numerically. Lodato \& Rice (2004, 2005) studied angular momentum transport in a model system composed of an isolated disk that cools everywhere on a time scale that is a fixed multiple of the local dynamical time. They found that the analytic estimate for the stress, derived from the assumption of local thermal balance, was a good match to the numerical results provided that the disk mass was not too high, $M_{\text {disk }} / M_{*} \lesssim 0.25$. A more quantitative analysis was carried out by Cossins, Lodato \& Clarke (2009), who explicitly evaluated the pattern speed of the waves generated by self-gravity within a disk described using a similarly simple prescription for the cooling time. For $M_{\text {disk }} / M_{*}=0.1$, they bounded departures from local behavior at the $10 \%$ level. Broadly similar results also hold for more realistic disks, in which the cooling time scale, set by the radiative physics, is a strong function of radius. Boley et al. (2006) used a radiation hydrodynamics code to study the evolution of a moderately massive disk $\left(M_{\text {disk }} / M_{*}=0.14\right)$ around a low mass star. They found that, although some non-local transport did occur, the predictions of a local model based upon the cooling time were consistent with the simulation across a substantial region of the disk. Likewise, simulations including radiative transfer by Forgan et al. (2010) showed significant non-local effects only when $M_{\text {disk }} / M_{*} \gtrsim 0.5$.

The conditions for fragmentation of a low-mass self-gravitating disk can also be written, to a first approximation, as a function of local disk conditions. Gammie (2001), using local numerical simulations, found that if the cooling could be parameterized as,

$$
t_{\mathrm{cool}}=\beta \Omega_{\mathrm{K}}^{-1},
$$


then for $\beta \lesssim 3$ fragmentation followed promptly as soon as the disk cooled to low $Q$. Short cooling times allow the disk to radiate away the energy provided by shocks or turbulent dissipation, such that neither pressure support nor shear suffice to avert gravitational collapse (Shlosman \& Begelman 1987). The thermal condition for fragmentation in this simple form applies strictly only in the limit where the cooling time in the disk is locally constant. Using a more realistic model, in which the cooling was calculated using a one-zone vertical model that took account of the optical depth, Johnson \& Gammie (2003) found that strong variations in the opacity with temperature could result in large changes to the critical cooling time scale for fragmentation. In practice, however, the opacity in protoplanetary disks is a (relatively) slowly varying function for the temperatures, below the dust sublimation temperature $T \approx 1500 \mathrm{~K}$, that are most likely to coincide with self-gravitating regions. Large deviations from the predictions of constant cooling time models are thus only likely for very high accretion rates $\dot{M} \gtrsim 10^{-5} M_{\odot} \mathrm{yr}^{-1}$ (Cossins, Lodato \& Clarke 2010).

It is unclear how accurately the local thermal criterion for fragmentation - derived from two dimensional simulations - holds in three dimensions in global disks. Early global simulations found surprisingly good quantitative agreement. Rice et al. (2003a), using Smooth Particle Hydrodynamics (SPH) simulations, found that for low-mass disks (roughly those for which $M_{\text {disk }} / M_{*} \sim 0.1$, or less), the condition to avoid fragmentation could indeed be expressed via a minimum cooling time, given for $\gamma=5 / 3$ by $\beta \approx 5$. Equivalently (if irradiation is not important) disks can stably transport angular momentum in a self-regulated state without fragmenting up to a maximum $\alpha \simeq 0.1$ (Rice, Lodato \& Armitage 2005). Similarly, Mejía et al. (2005), using a grid-based hydrodynamics code, did not obtain fragmentation for a cooling time $\beta \approx 6$. Recent higher resolution SPH simulations, however, see evidence for fragmentation at significantly longer (by a factor of two) cooling times (Meru \& Bate 2010). Additional work, ideally using independent numerical techniques, is needed to clarify the relation between the two dimensional local and fully three dimensional fragmentation boundary.

The numerical results imply that for a low mass disk both the angular momentum transport efficiency of a stable self-gravitating disk, and the conditions for fragmentation, can be written as functions of the angular velocity and local opacity. It is then possible to abstract the physics of disk self-gravity into a one-dimensional model (identical in spirit to that of Lin \& Pringle 1987), in which $\nu(r, \Sigma)$ is completely determined by the requirement of thermal equilibrium, and seek either steady-state analytic (Clarke 2009, Cossins, Lodato \& Clarke 2010; Matzner \& Levin 2005; Rafikov 2009) or time-dependent numerical solutions (Rice \& Armitage 2009; Zhu, Hartmann \& Gammie 2010). A central result of such simplified models is that fragmentation cannot occur in the inner disk (Rafikov 2005). Matzner \& Levin (2005), for example, derived a critical angular velocity for fragmentation of $\Omega_{\mathrm{frag}} \simeq 4.3 \times 10^{-10} \mathrm{~s}^{-1}$, corresponding to a radius of $60 \mathrm{AU}$ around a Solar-mass star. Results from a similar calculation are plotted in Figure 3, which shows the region in the $(r, \dot{M})$ plane for which a non-irradiated self-gravitating disk would be unable to maintain self-regulated transport, and might fragment. In agreement with numerous authors (Clarke 2009; Cossins, Lodato \& Clarke 2010; Rafikov 2009, Zhu, Hartmann \& Gammie 2010), we find that disks with $\dot{M} \gtrsim 10^{-7} M_{\odot} \mathrm{yr}^{-1}$ can be unstable to fragmentation (or, some other breakdown of local behavior, such as bursts) at radii beyond $50-10^{2} \mathrm{AU}$, but are stable closer in. This result is consistent with several simulations of protoplanetary disks that include radiative transfer (e.g. Boley et al. 2010, Stamatellos \& Whitworth 2008), as expected given that the assumptions underlying the analytic theory were derived numerically. Some other radiation hydrodynamic simulations, however, obtain fragmentation at significantly smaller radii $r \sim 10$ AU (Boss 2008, Mayer et al. 2007). The reason for these discrepant results is not fully understood, although differences in the treatment of the chemical composition (e.g. the temperature dependence of $\mu$ ) and radiative transfer may play a role (Cai et al. 2010).

In the context of star formation, $50-10^{2} \mathrm{AU}$ is not a particularly large radius, and the analytic result then suggests molecular cloud cores with sufficiently high specific angular momentum would 


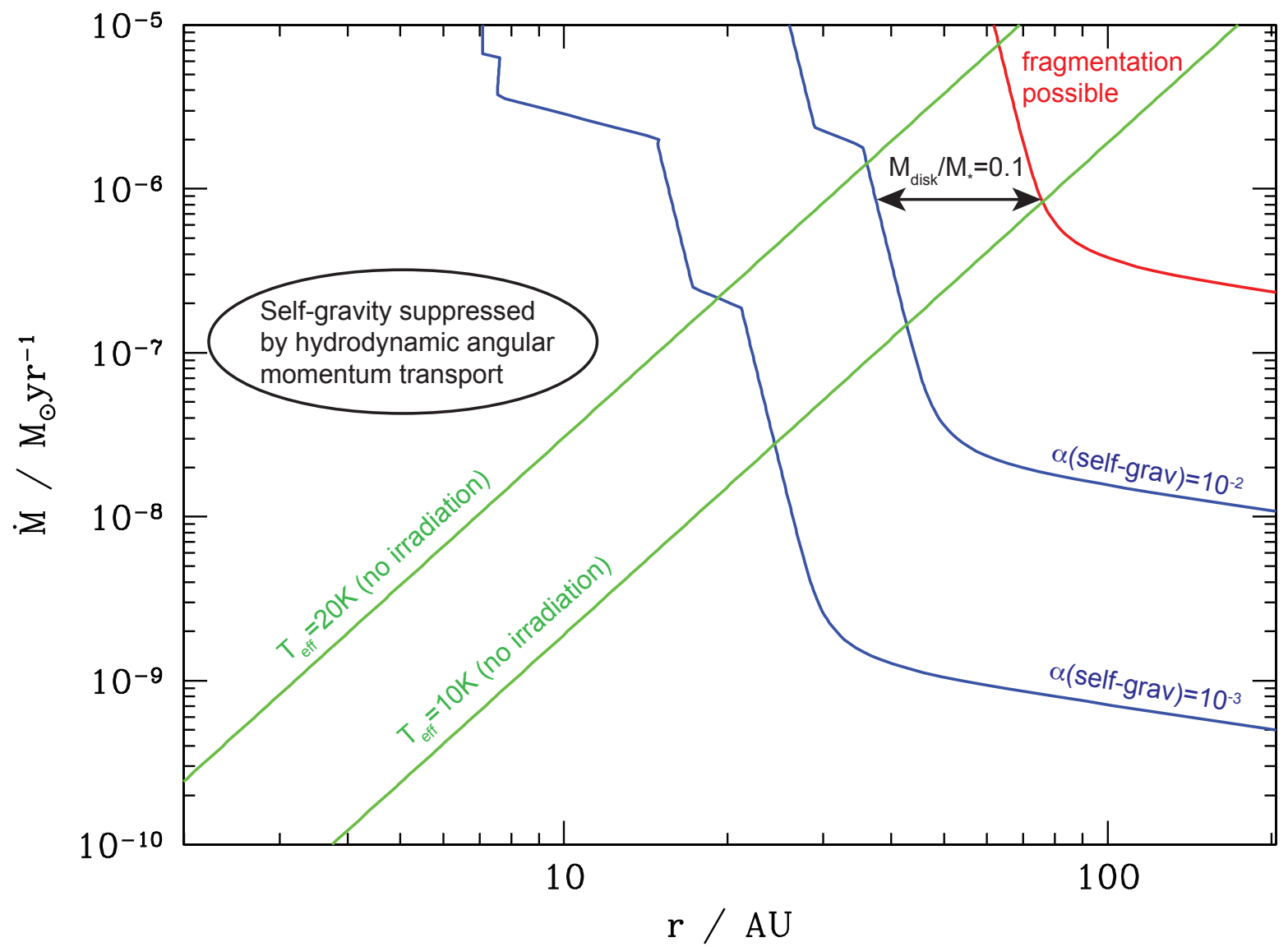

Figure 3: The structure of a steady-state self-gravitating disk around a $1 M_{\odot}$ star, calculated assuming that self-gravity acts as a local angular momentum transport process whose efficiency is set by the requirement of thermal equilibrium (Clarke 2009, Rafikov 2009). The opacity includes contributions from water ice, amorphous carbon, silicates and graphite (Z. Zhu, private communication). If self-gravity is the sole source of angular momentum transport, the effective $\alpha_{\text {grav }}$ generated by the self-gravitating turbulence is an increasing function of radius, and there is a maximum radius (shown as the red line) beyond which fragmentation is predicted to occur. The blue curves show radii where $\alpha_{\text {grav }}=10^{-2}$ and $10^{-3}$. If other sources of angular momentum transport co-exist with self-gravity, and generate stresses of this magnitude, these radii denote the approximate inner edge of the self-gravitating disk. The green lines show where the effective temperature of the luminous disk equals $10 \mathrm{~K}$ or $20 \mathrm{~K}$; if an external irradiation field heats the disk it would affect the dynamics to the right of the green lines.

be susceptible to fragmentation at their outer edge (Kratter, Murray-Clay \& Youdin 2010; Matzner \& Levin 2005; Rice, Mayo \& Armitage 2010; Stamatellos, Hubber \& Whitworth 2007; Stamatellos \& Whitworth 2009). There are, however, two caveats. First, the effective temperature of an isolated self-gravitating disk at the innermost fragmenting radius is quite low, typically around 10-20 K (Matzner \& Levin 2005). This implies that the dynamics of self-gravitating disks in the potentially fragmenting region are sensitive to modest levels of external irradiation, which will tend to weaken gravitational instabilities (Cai et al. 2008, Stamatellos \& Whitworth 2008, Vorobyov \& Basu 2010). Second, the innermost fragmenting radius is calculated for a given opacity, and will 
vary with the metallicity of the disk and as a result of particle coagulation (Cai et al. 2006). For an opacity appropriate for ice grains, for example, $\kappa=\kappa_{0} T^{2}$ (Semenov et al. 2003), and $\Omega_{\text {frag }} \propto \kappa_{0}^{-1 / 3}$ (Matzner \& Levin 2005, Rafikov 2009). Reduced values of the opacity would therefore push the fragmentation boundary interior to the fiducial radius of $50-10^{2} \mathrm{AU}$.

Immediately interior to the radius where $\Omega_{\mathrm{K}}=\Omega_{\mathrm{frag}}$, the strength of self-gravitating transport is close to the maximum value, $\alpha \approx 0.1$. At smaller radii the disk becomes increasingly optically thick, and the longer cooling time results in lower values of $\alpha$ (Equation 13). This means that the surface density in self-gravitating disks at small radii is high, and that even very weak transport by other mechanisms will suffice to render the disk stable against self-gravity. Curves corresponding to a self-gravitating $\alpha=10^{-2}$ and $10^{-3}$ are shown in Figure 3, from which it is clear that is hard to sustain a strongly self-gravitating disk much interior to $10 \mathrm{AU}$. This implies that, although shocks in a strongly self-gravitating disk are a candidate mechanism for chondrule formation (Boss \& Durisen 2005), it is hard to construct self-consistent models in which strong enough shocks would be present at small radii (Boley \& Durisen 2008).

For more massive isolated disks, the nature of the departure from local behavior is reasonably well-established. As the mass ratio between the disk and star increases, there is an increasing domination of low-order global spiral modes (illustrated in Figure 4), and a transition to timedependent "bursting" behavior (Vorobyov \& Basu 2006). For protostellar disks at early times, however, multiple confounding factors come into play, including irradiation from the envelope, and dynamical effects associated with the addition of mass (Harsono, Alexander \& Levin 2010), angular momentum, and thermal energy from infall. All of these will affect the self-gravitating evolution.

Kratter et al. (2010) simulated the evolution of protostellar disks formed from rapid infall, assuming that the disk behaves isothermally prior to fragmentation. The assumption of isothermality precludes comparison with the results for low mass, isolated disks - where thermal physics is of primary importance - though it represents a reasonable starting point for the background structure of disks whose thermal balance is dominated by external irradiation (Krumholz, Klein \& McKee 2007). Kratter et al. (2010) express the rate of infall via a parameter,

$$
\xi=\frac{\dot{M}_{\text {in }} G}{c_{s}^{3}}
$$

where $\dot{M}_{\text {in }}$ is the rate of infall onto the disk and $c_{s}$ is the disk sound speed. Since the rate of accretion through a self-gravitating disk can be written as $\dot{M}=3 \alpha c_{s}^{3} /\left(G Q_{0}\right)$, the parameter $\xi$ represents the infall rate scaled to the maximum (at $\alpha \sim 1$ ) accretion rate that a self-gravitating disk might be expected to sustain. Numerically, it is found that disks are able to avoid fragmentation for $\xi \lesssim \xi_{\max }$, where $\xi_{\max }$ varies with the angular momentum of the infalling gas but is typically $\xi_{\max } \sim 2-3$ (Kratter et al. 2010). The corresponding $\alpha$, averaged over the disk, is $\alpha \approx 1$, significantly higher than the maximum value for an isolated low-mass disk. Accretion in this regime is highly variable, due to a combination of the dominance of global modes (Forgan et al. 2010, Laughlin \& Bodenheimer 1994), the effect of infall, and the formation and disruption of clumps (Boley et al. 2010, Vorobyov \& Basu 2010). Disks can attain masses as high as $M_{\text {disk }} \approx M_{*}$ while remaining in the stable regime; yet more massive disks invariably fragment.

\subsection{Magnetorotational Instability}

An accretion disk coupled dynamically to a weak magnetic field is subject to instabilities that initiate and sustain MHD turbulence, which in turn generates Maxwell and Reynolds stresses that result in outward angular momentum transport. In ideal MHD (i.e. when terms associated with Ohmic and ambipolar diffusion, and with the Hall effect, can be neglected), the conditions necessary for this instability - known as the magnetorotational instability (MRI; Balbus \& Hawley 1991) - are 


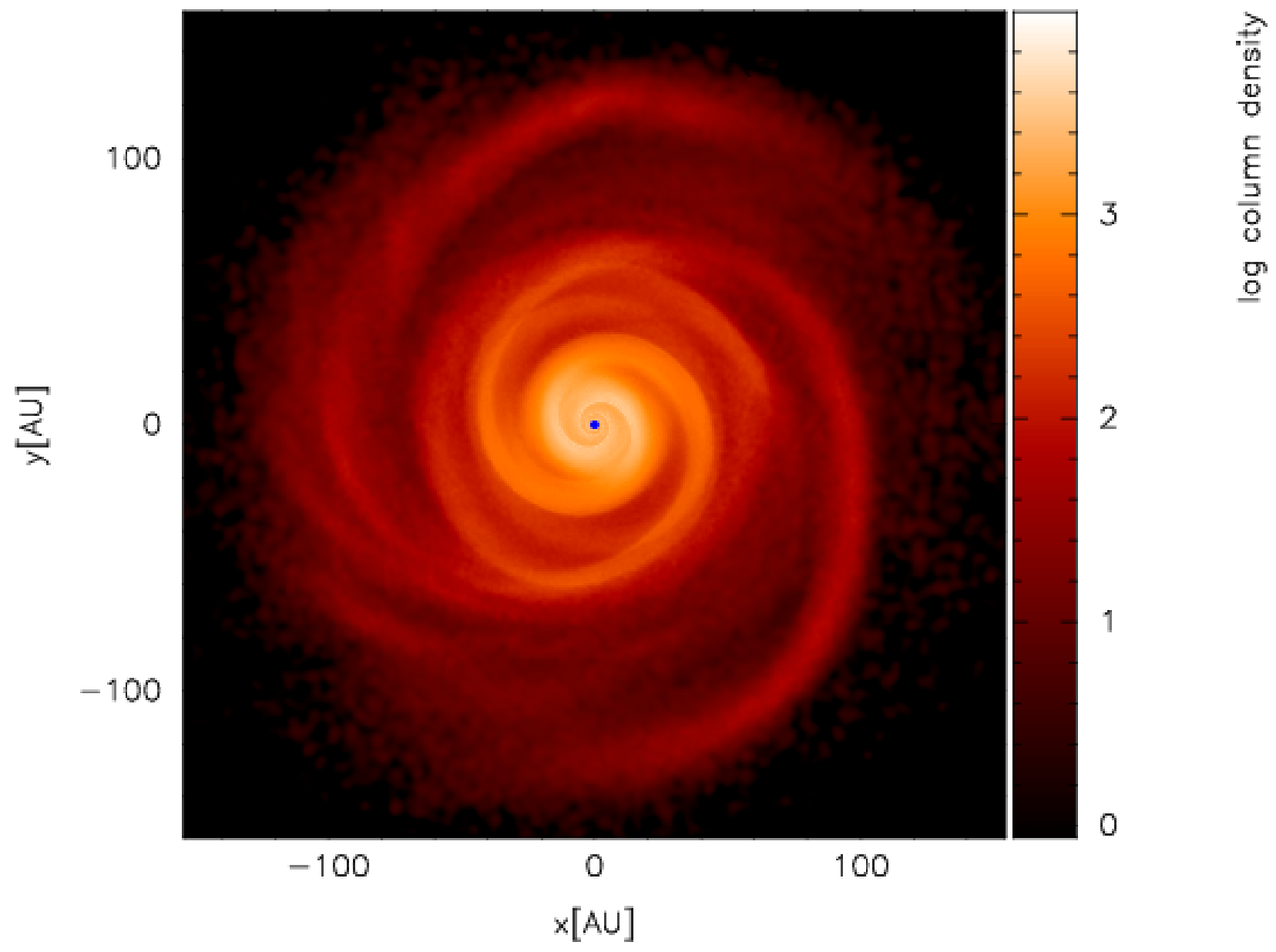

Figure 4: Structure of a massive $\left(M_{\text {disk }}=M_{*}=M_{\odot}\right)$ self-gravitating disk in the non-fragmenting regime, based on simulations by Forgan et al. (2010). The simulated disk had an initial surface density profile $\Sigma \propto r^{-3 / 2}$, and was evolved with an approximate radiative transfer scheme. At this mass ratio, strong low-order spiral structure dominates.

simply stated (for an excellent review, see Balbus \& Hawley 1998). A disk threaded by a vertical magnetic field, that is "weak" in the sense that,

$$
\frac{B_{z}^{2}}{8 \pi} \lesssim \frac{3}{\pi^{2}} \rho c_{s}^{2},
$$

is subject to a local, linear instability, provided that,

$$
\frac{\mathrm{d}}{\mathrm{d} r}\left(\Omega^{2}\right)<0
$$

Keplerian disks are therefore always unstable to the MRI, unless they are threaded by strong magnetic fields whose energy density exceeds the thermal energy within the disk (even then, the MRI could arise as a secondary instability if the strong field is unstable to, say, Parker instability; Johansen \& Levin 2008). Although there is no observational proof, the ubiquity of the conditions necessary for the MRI, together with its fast growth rate ( $\sim \Omega$ for the fastest growing mode), strongly suggest that the MRI is the source of angular momentum transport in non-self-gravitating disks around white dwarfs, neutron stars, and black holes. 
For protoplanetary disks, no comparably definitive statement is possible. The gas in protoplanetary disks is cool, dense, and overwhelmingly neutral, and as a result non-ideal MHD effects are important. Complicating matters further, different non-ideal terms dominate at different $(r, z)$ within the disk. The strength and nature of turbulence sustained by the MRI is then not expected to be universal, but rather must be calculated as a function of the local physical conditions. (It is also possible that some disks might be threaded by magnetic fields that are too strong to admit the MRI altogether.)

Although the full problem is complex, considerable insight can be gained by examining a toy model in which the sole non-ideal effect is Ohmic resistivity (the discussion here is adapted from Gammie 1996). The induction equation has the form,

$$
\frac{\partial \mathbf{B}}{\partial t}=\nabla \times[\mathbf{v} \times \mathbf{B}-\eta \nabla \times \mathbf{B}]
$$

where $\eta$, the magnetic resistivity, enters as a diffusive term that acts to smooth out small scale structure in the magnetic field. To estimate the effect of resistivity on the MRI, we note that the time scale for the growth of the MRI on a scale $\lambda$ in ideal MHD is $\tau_{\text {MRI }} \sim \lambda / v_{A}$, where $v_{A}$, the Alfvén velocity, is given by $v_{A}=B / \sqrt{4 \pi \rho}$. At the same scale, the time scale for resistive damping is $\tau_{\eta} \sim \lambda^{2} / \eta$. Equating these time scales at $\lambda=h$ (which is where the effects of non-zero $\eta$ are weakest), we find that to suppress the growth of the MRI requires,

$$
\eta \gtrsim h v_{A} \text {. }
$$

Defining the magnetic Reynolds number, $\operatorname{Re}_{M}=h v_{A} / \eta$, an equivalent statement is that the MRI will not exist for $\operatorname{Re}_{M} \lesssim 1$ (note that care is needed when reading the literature, since at least three related but distinct definitions of $\operatorname{Re}_{M}$ are in common currency).

Next, we estimate the electron fraction needed to attain $\operatorname{Re}_{M}=1$ under disk conditions. In a gas composed of electrons and neutral particles, collisions between the two species limit the conductivity and yield a resistivity that is given by (Blaes \& Balbus 1994, Spitzer 1962),

$$
\eta=234\left(\frac{n}{n_{e}}\right) T^{1 / 2} \mathrm{~cm}^{2} \mathrm{~s}^{-1},
$$

where $n$ and $n_{e}$ are the number densities of the neutral species and electrons, respectively. For a magnetic field strength such that $v_{A}=\epsilon c_{s}$, the critical electron fraction necessary to reach $\operatorname{Re}_{M}=1$ at $1 \mathrm{AU}$ can be estimated as,

$$
\frac{n_{e}}{n} \sim 5 \times 10^{-13}\left(\frac{h / r}{0.05}\right)^{-1}\left(\frac{\epsilon}{0.1}\right)^{-1} .
$$

This is a very small number. It is, however, much larger than the ionization fraction for disk gas in thermal equilibrium at a few hundred K. Apart from very close to the star (where thermal ionization of alkali metals provides sufficient ionization), the operation of the MRI in protoplanetary disks therefore depends on non-thermal ionization processes. For a particularly simple example, we can evaluate the electron fraction expected if the dominant source of ionization is the decay of ${ }^{26} \mathrm{Al}$. To an order of magnitude, the expected ionization rate is $\zeta=10^{-19} \mathrm{~s}^{-1}$ (Stepinski 1992). Balancing this against dissociative recombination in the gas phase, we find,

$$
\frac{n_{e}}{n}=\sqrt{\frac{\zeta}{\beta n}} \simeq 10^{-13}\left(\frac{\zeta}{10^{-19} \mathrm{~s}^{-1}}\right)^{1 / 2}\left(\frac{T}{500 \mathrm{~K}}\right)^{1 / 4}\left(\frac{n}{10^{14} \mathrm{~cm}^{3}}\right)^{-1 / 2},
$$

where we have assumed a rate coefficient $\beta=3 \times 10^{-6} T^{-1 / 2} \mathrm{~cm}^{3} \mathrm{~s}^{-1}$ (e.g. Fromang, Terquem \& Balbus 2002), and substituted parameters appropriate for the disk mid-plane near 1 AU. 
Comparing Equations (23) and (24) shows why determining the efficacy of the MRI in protoplanetary disks is a hard problem; the electron fraction that is expected to be present due to non-thermal ionization can be of the same order of magnitude as the simple estimate of the critical fraction needed to sustain the MRI. Obtaining the right answer requires detailed calculations that address each of the places where we resorted to order of magnitude arguments above:

1. What is the effect of non-ideal terms on the MRI? Specifically, how do Ohmic and ambipolar diffusion, and the Hall effect, alter the linear and non-linear development of MRI-driven disk turbulence?

2. What is the spatial distribution of ionization within the disk, due to non-thermal processes including X-ray irradiation, cosmic rays, and radioactive decay?

3. What is the recombination rate, due to both gas phase and surface processes (on dust grains)?

Despite substantial progress on each of these individual issues, discussed below, the compounded uncertainty involved when they are combined precludes a definitive assessment of how efficient the MRI is at transporting angular momentum in protoplanetary disks. The implicit coupling to the physics of dust coagulation, that enters via the influence of dust on the recombination rate, is particularly troubling, as dust coagulation is subject to numerous additional uncertainties.

3.2.1 EFFECTS OF NON-IDEAL TERMS The evolution of the magnetic field within a weakly ionized protoplanetary disk obeys an induction equation that reads,

$$
\frac{\partial \mathbf{B}}{\partial t}=\nabla \times\left[\mathbf{v} \times \mathbf{B}-\eta \nabla \times \mathbf{B}-\frac{\mathbf{J} \times \mathbf{B}}{e n_{e}}+\frac{(\mathbf{J} \times \mathbf{B}) \times \mathbf{B}}{c \gamma \rho_{i} \rho}\right] .
$$

Here $\mathbf{v}$ is the velocity of the dominant neutral component of the fluid, with density $\rho$, the current $\mathbf{J}=c(\nabla \times \mathbf{B}) / 4 \pi$, and $\rho_{i}$ is the density of ions. $\gamma$, the drag coefficient, is given in terms of the ion-neutral collision rate $\langle\sigma \omega\rangle_{i}$ by $\gamma=\langle\sigma \omega\rangle_{i} /\left(m_{i}+m_{n}\right)$, where $m_{i}$ and $m_{n}$ are the masses of the ions and neutrals respectively (for a derivation see, e.g. Balbus 2010). The terms on the right-hand-side describe magnetic induction (the frozen-in field behavior of ideal MHD) and the three non-ideal effects, Ohmic diffusion (denoted as $\mathrm{O}$ ), the Hall effect $(\mathrm{H})$, and ambipolar diffusion (A). Physically, ambipolar diffusion is dominant when the field is well-coupled to the ions and electrons, such that the field drifts with the charged species relative to the neutral component. Ohmic diffusion dominates when the conductivity is so low that the field is imperfectly coupled to both the electrons and the ions. Finally, the Hall effect is most important in an intermediate regime where the field is well-coupled to the electrons but not to the ions.

Determining the absolute importance of the non-ideal terms (i.e. their ratios to the inductive term) requires solving for the ionization state of the disk. As we have already observed, this is difficult everywhere except in the very innermost regions, interior to about $0.1 \mathrm{AU}$, where thermal ionization dominates. It is much easier to assess the relative magnitude of the non-ideal terms, which depend only upon the temperature, $T$, and total number density $n$. Balbus \& Terquem (2001) estimate these ratios by assuming that electrons and singly-ionized ions are the charge carriers, that the typical fluid velocities are $\sim v_{A}$, the Alfvén speed, and that typical gradients are $\sim h^{-1}$. They obtain,

$$
\begin{aligned}
\frac{\mathrm{O}}{\mathrm{H}} & =\left(\frac{n}{8 \times 10^{17} \mathrm{~cm}^{-3}}\right)^{1 / 2}\left(\frac{v_{A}}{c_{s}}\right)^{-1} \\
\overline{\mathrm{H}} & =\left(\frac{n}{9 \times 10^{12} \mathrm{~cm}^{-3}}\right)^{-1 / 2}\left(\frac{T}{10^{3} \mathrm{~K}}\right)^{1 / 2}\left(\frac{v_{A}}{c_{s}}\right) .
\end{aligned}
$$

Using these expressions, we show in Figure 5 the relative importance of the three non-ideal effects as a function of density and temperature (after Kunz \& Balbus 2004). Over-plotted on the Figure 


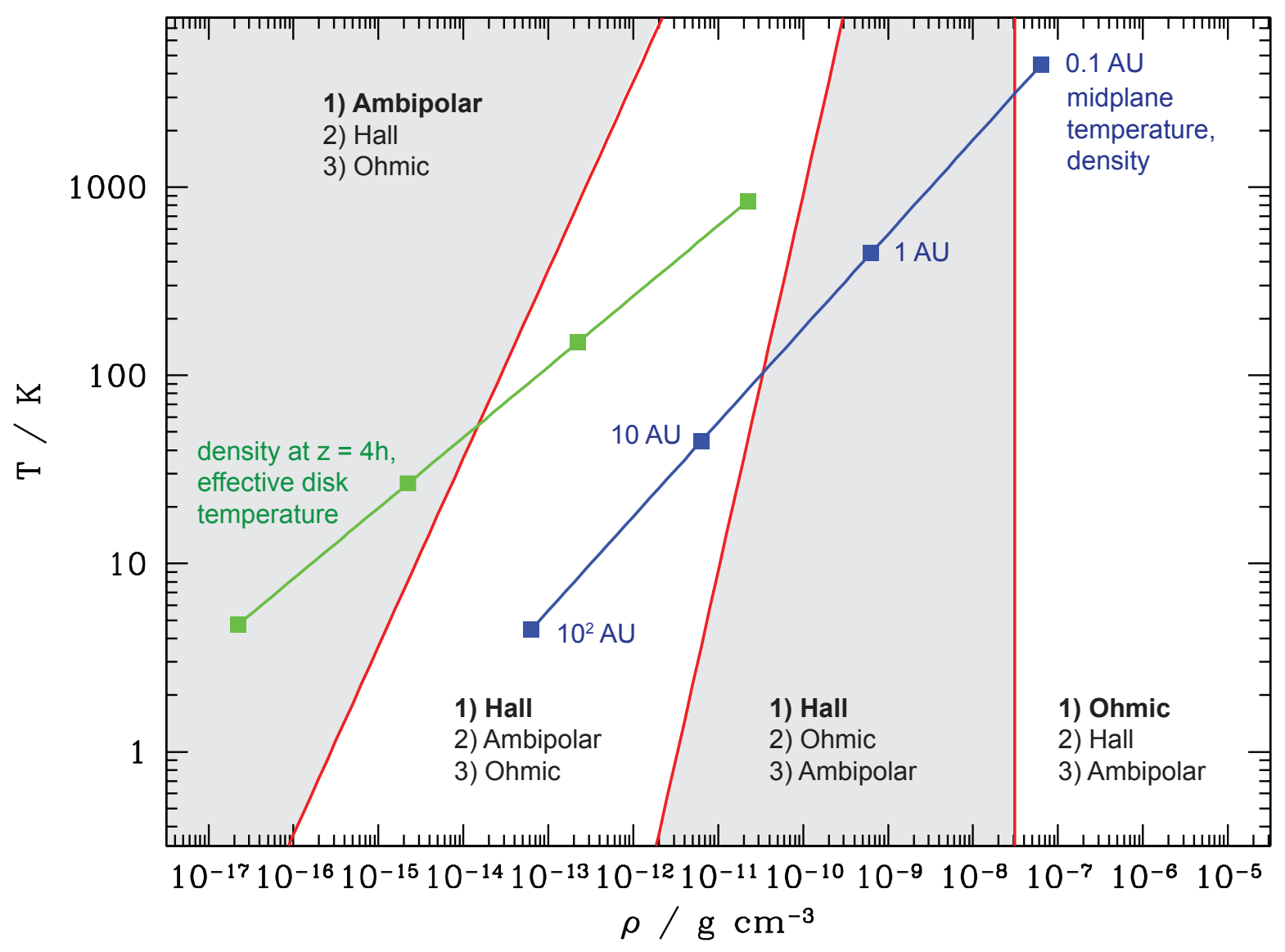

Figure 5: The relative importance of non-ideal MHD terms is shown in the $(\rho, T)$ plane (Balbus \& Terquem 2001, Kunz \& Balbus 2004), assuming a magnetic field strength such that $v_{A} / c_{s}=0.1$. Also plotted are very approximate tracks showing the radial variation of physical conditions at the mid-plane, and near the surface, of protoplanetary disks. The mid-plane conditions are estimated for a disk around a Solar mass star with $\Sigma=10^{3}(r / 1 \mathrm{AU})^{-1} \mathrm{~g} \mathrm{~cm}^{-2}$, and $(h / r)=0.04$. The surface conditions are estimated from the density at $z= \pm 4 h$ (using a gaussian density profile), assuming that the temperature is the effective temperature for a steady-state disk accreting at $\dot{M}=10^{-7} M_{\odot} \mathrm{yr}^{-1}$.

are order of magnitude estimates of the run of density and temperature within a disk, both at the mid-plane and near the surface. At the disk mid-plane, a combination of the Hall effect and Ohmic diffusion are likely to be the most important non-ideal terms interior to $\sim 10 \mathrm{AU}$. At larger mid-plane radii - and in the disk atmosphere at all radii - the Hall effect and ambipolar diffusion are dominant.

The linear stability of disks to growth of the MRI has been studied for each of the relevant combinations of non-ideal terms. The simplest case is the purely resistive limit, analyzed by Jin (1996), whose mathematical behavior closely matches that expected from the heuristic arguments given above. If Ohmic diffusion is the sole non-ideal effect, the growth of the MRI is damped whenever $\operatorname{Re}_{M} \lesssim 1$ (Jin 1996, Sano \& Miyama 1999). More typically, however, Ohmic diffusion will be accompanied by a strong Hall effect, which qualitatively modifies the disk's linear stability 
(Balbus \& Terquem 2001, Wardle 1999). Most strikingly, for a Keplerian disk in which $\kappa=\Omega_{\mathrm{K}}$, if

$$
\operatorname{Re}_{M}^{-1}<\frac{3}{4}\left(\frac{v_{A}}{c_{s}}\right)
$$

i.e. for disks in which Ohmic diffusivity is not extremely strong, the Hall effect destabilizes some perturbations on arbitrarily small scales even in the presence of non-zero $\eta$. Other perturbations are stabilized. In general, for a perturbation with wavevector $\mathbf{k}$, the Hall term acts to stabilize or destabilize depending upon the sign of $(\mathbf{k} \cdot \boldsymbol{\Omega})(\mathbf{k} \cdot \mathbf{B})$ (Balbus \& Terquem 2001). If vertical fields thread the disk, one may conjecture that the resulting asymmetry in the dynamics - between fields that are aligned or anti-aligned with the angular momentum vector - could have physical consequences.

In addition to the Hall / Ohmic-dominated regime, the case where ambipolar diffusion is the strongest non-ideal effect may also be relevant to protoplanetary disks. The linear physics of the MRI in this limit has been analyzed using a two-fluid model by Blaes \& Balbus (1994), and in a single-fluid approximation by Kunz \& Balbus (2004) and by Desch (2004). The novel feature of the ambipolar MRI is that - even in the absence of the Hall effect - the addition of what is an apparently dissipative term can destabilize fluid configurations that would be stable in purely ideal MHD (Kunz \& Balbus 2004). Nevertheless, it remains true that the growth rate of the ambipolar MRI is strongly suppressed once the ion-neutral collision time scale exceeds $\Omega_{\mathrm{K}}^{-1}$. As with Ohmic diffusion, one can therefore define an "ambipolar Reynolds number", $\operatorname{Re}_{A}=\gamma \rho_{i} / \Omega_{\mathrm{K}}$, and express the condition for efficient MRI growth simply as requiring $\operatorname{Re}_{A} \gtrsim 1$.

3.2.2 Non-linear MRI Evolution The linear physics of the MRI, in the various nonideal regimes, plausibly defines a set of necessary conditions for the onset of MHD turbulence and angular momentum transport. It is less clear whether linear instability generally defines sufficient conditions for sustained transport, since, for a disk with zero net magnetic flux, no conservation law prohibits the disk from reconnecting its initial field and thereby accessing a hydrodynamically stable, field-free state. (This curious behavior is seen in MHD simulations in small domains that omit explicit dissipation, e.g. Fromang \& Papaloizou 2007; Guan et al. 2009; Pessah, Chan \& Psaltis 2007; Simon, Hawley \& Beckwith 2009). Numerical simulations to study the non-linear phase can be carried out in either a local ("shearing box") geometry, which amounts to modeling a small patch of disk in a co-rotating co-ordinate system (Hawley, Gammie \& Balbus 1995), or globally (Armitage 1998). A secondary distinction is between unstratified simulations, which by omitting the vertical component of gravity have no mean vertical density gradient (this is done for reasons of computational economy), and stratified calculations (Brandenburg et al. 1995, Stone et al. 1996) that are required in order to model physical effects such as buoyancy.

A tentative understanding of the non-linear behavior of the MRI has been attained only in the ideal MHD limit. In this regime, stratified local simulations confirm that the MRI yields sustained MHD turbulence, in which outward angular momentum transport occurs primarily as a result of Maxwell stresses (though fluid stresses also contribute at a non-negligible level). The toroidal magnetic field is dominant, and displays quasi-periodic cyclic behavior near the mid-plane on a time scale of $\sim 10$ orbits (Brandenburg et al. 1995; Davis, Stone \& Pessah 2010; Shi, Krolik \& Hirose 2010; Stone et al. 1996). Associated with this cycle, magnetic field rises buoyantly through the disk, forming a "butterfly diagram" in two-dimensional plots of $B_{\phi}(z, t)$ that is strikingly reminiscent of Solar dynamo cycles. Typical values of $\alpha$ in zero net field simulations, averaged over long time scales, are $\alpha \sim 10^{-2}$ (Davis, Stone \& Pessah 2010; Shi, Krolik \& Hirose 2010). Larger values are possible if a net field threads the disk, in which case unstratified simulations show that the stress scales with $\beta$, the ratio of gas pressure to the magnetic pressure associated with the net flux, as $\alpha \propto \beta^{-1 / 2}$ (Hawley, Gammie \& Balbus 1995). Some stratified simulations suggest that a net field may also promote mass loss via a disk wind (Suzuki \& Inutsuka 2009).

Although the basic results summarized above appear robust, loose ends of unknown physical 
significance are present even in "almost ideal" MHD, where viscosity and resistivity are introduced solely to give well-controlled dissipation at the grid scale. In particular, the saturation level (and even the persistence) of turbulence has been observed to depend upon the magnetic Prandtl number $\mathrm{Pm}=\nu / \eta$, even at relatively high Reynolds numbers where Ohmic damping of linear modes should not be playing a role (Fromang et al. 2007, Longaretti \& Lesur 2010, Simon \& Hawley 2009). Whether this dependence persists at the Reynolds numbers of real disks (which are vastly larger than those accessible computationally) remains an open question.

Moving beyond ideal MHD, exploratory local simulations have studied the non-ideal regimes relevant to protoplanetary disks. Caution is required in interpreting many of the results, since the bulk of the work predates the realization that unstratified local simulations with zero-net flux exhibit pathological convergence properties. Nonetheless, simulations that include Ohmic diffusion confirm the analytic expectation of a critical Reynolds number below which MRI activity is quenched (Fleming, Stone \& Hawley 2000; Sano \& Inutsuka 2001), and suggest that the primary criterion for quenching continues to be set by the resistivity even in the presence of the Hall effect (Sano \& Stone 2002). Simon \& Hawley (2009), for example, quantified the conditions necessary for MRI activity in unstratified local simulations with a net toroidal field, including both resistivity and an explicit microphysical viscosity. They found that turbulence decayed when a dimensionless measure of the importance of resistivity, the Elsasser number,

$$
\Lambda \equiv \frac{v_{A}^{2}}{\eta \Omega_{\mathrm{K}}} \lesssim 10^{2} .
$$

For somewhat weaker resistivity, additional time-dependent behavior, over and above that seen in ideal MHD, develops (Simon, Hawley \& Beckwith 2010). A cycle develops in which the mid-plane MRI is first quenched, but subsequently reignites due to long time scale growth of the toroidal magnetic field. Lastly, in the ambipolar regime, numerical work by Mac Low et al. (1995) and by Hawley \& Stone (1998) shows that the MRI is suppressed for ion-neutral collision frequencies below about $10^{2} \Omega_{\mathrm{K}}$.

By any accounting our quantitative understanding of the non-ideal MRI is incomplete, and absent higher resolution calculations the quoted thresholds for MRI activity in disks subject to Ohmic / Hall or ambipolar effects should be regarded as preliminary. However, even a definitive local determination of the conditions necessary for the non-ideal MRI to operate would address only part of the broader physical problem. The sustenance of MHD turbulence in a local simulation depends upon the strength and geometry of the field threading the volume, which in more complete disk models would be specified by external factors: either the initial conditions of disk formation or global magnetic linkage between different regions of the disk (Sorathia, Reynolds \& Armitage 2010; Tout \& Pringle 1996; Uzdensky \& Goodman 2008). Global numerical simulations (Fromang \& Papaloizou 2006) that include non-ideal processes (Dzyurkevich et al. 2010) are currently at an early stage of development, but will be important for studying the evolution of magnetic field both within and above the disk plane.

3.2.3 IONIZATION AND RECOMBINATION PROCESSES The absolute importance of the nonideal MHD terms depends upon the conductivity of the gas, and hence on the ionization fraction, $x=n_{e} / n$ (e.g. Equation 22 for $\eta$ ). Collisional ionization of trace species, primarily potassium, suffices to yield $x \sim 10^{-12}$ at $T \simeq 10^{3} \mathrm{~K}$, so gas temperatures above this limit are likely enough to assure MRI activity. In $\alpha$ models of protoplanetary disks (Bell et al. 1997), a mid-plane temperature of $10^{3} \mathrm{~K}$ corresponds to a radius between $\approx 0.1 \mathrm{AU}$ (for a disk accreting at $10^{-9} M_{\odot} \mathrm{yr}^{-1}$, with $\alpha=10^{-2}$ ), and $\approx 5 \mathrm{AU}$ (at $10^{-5} M_{\odot} \mathrm{yr}^{-1}$ ). Gas in the innermost disk, including that interacting directly with the stellar magnetosphere (Königl 1991), can thus be assumed to be MRI-active without recourse to further calculations.

At larger radii in the disk, the collisional ionization fraction is for all practical purposes zero, and $x$ must instead be computed by balancing non-thermal sources of ionization with recombinations. 
Although the non-thermal power required to maintain $x$ above the threshold for MRI activity is modest (Inutsuka \& Sano 2005), the only indubitable source of significant ionization is disk irradiation by stellar X-rays, whose strength is well-determined observationally. In the Taurus star-forming region, for example, Güdel et al. (2007) find that the X-ray luminosity of detected sources scales with the stellar mass as,

$$
\log \left(L_{X} / \mathrm{erg} \mathrm{s}^{-1}\right)=1.54 \log \left(M_{*} / M_{\odot}\right)+30.31
$$

for masses in the range $0.1 M_{\odot} \lesssim M_{*}<2 M_{\odot}$. The spectral properties are complex, but a simple representation, used in an analysis of Orion sources by Preibisch et al. (2005), combines a "cool" thermal plasma component at $T \approx 10^{7} \mathrm{~K}$, with a hotter component at $T \approx 3 \times 10^{7} \mathrm{~K}$. X-ray photoionization calculations (Glassgold, Najita \& Igea 1997), and Monte Carlo simulations (Ercolano et al. 2008, Igea \& Glassgold 1999), show that these spectra and luminosities imply enough hard X-ray photons to ionize significant disk columns to interesting levels. An analytic form for the ionization rate $\zeta$ has been given by Turner \& Sano (2008), who find that the results of Igea \& Glassgold (1999) for a star emitting $5 \mathrm{keV}$ thermal X-rays at $L_{X}=2 \times 10^{30} \mathrm{erg} \mathrm{s}^{-1}$ can be fit as,

$$
\zeta(\Delta \Sigma)=2.6 \times 10^{-15}\left(\frac{r}{1 \mathrm{AU}}\right)^{-2} \exp \left[-\Delta \Sigma / 8.0 \mathrm{~g} \mathrm{~cm}^{-2}\right] \mathrm{s}^{-1}
$$

where $\Delta \Sigma$ is the column measured downward from the disk surface. This simple expression underestimates the ionization rate near the disk surface; a more detailed fit valid for lower column densities is provided by Bai \& Goodman (2009).

Figure 6 shows the vertical dependence of $\zeta$ implied by Equation (31), together with similarly rough estimates of the ionization rate that cosmic rays (Gammie 1996, Umebayashi \& Nakano 1981) and radioactive decay of short-lived isotopes (long-lived radionuclides can be neglected, Umebayashi \& Nakano 2009) might yield (Stepinski 1992). (Turner \& Drake 2009, have proposed that energetic stellar protons may also contribute a significant steady flux). The level of these other non-thermal sources of ionization is more uncertain than for stellar X-rays. In the Solar System, the magnetic field in the Solar wind serves to partially exclude cosmic rays (Spitzer \& Tomasko 1968), and the presumably more powerful stellar and disk winds of $\mathrm{T}$ Tauri stars may likewise shield the disk from the unattenuated cosmic ray flux. Similarly, isotopes such as ${ }^{26} \mathrm{Al}$ may largely be locked up into grains. Fortunately, the qualitative features of the ionization rate profile are largely independent of these unknowns. Generically, stellar X-rays will be the dominant ionization source near the disk surface, while the columns expected in disks ar $r \sim 1 \mathrm{AU}$ are more than large enough to shield the mid-plane from any reasonable ionizing flux of photons or particles. Even the maximum plausible ionization rate due to radioactive decay is small.

These uncertainties in the ionization rate pale in comparison to those afflicting estimates of the recombination rate. The obstacle here stems not from the physics of recombination, which is reasonably well-understood (for a discussion of the uncertainties due to disk chemistry, see e.g. Vasyunin et al. 2008), but rather from the fact that the answer depends sensitively on the unknown abundance of small dust grains. Before turning to this aspect of the problem, we first address the rate of recombination in dust-free gas. In this limit, the important reactions are dissociative recombination reaction with molecular ions, such as,

$$
\mathrm{HCO}^{+}+e^{-} \rightarrow \mathrm{CO}+\mathrm{H}
$$

radiative recombinations with metal ions,

$$
\mathrm{Mg}^{+}+e^{-} \rightarrow \mathrm{Mg}+\gamma
$$

and reactions which transfer charge from molecular to metal ions,

$$
\mathrm{HCO}^{+}+\mathrm{Mg} \rightarrow \mathrm{HCO}+\mathrm{Mg}^{+} \text {. }
$$




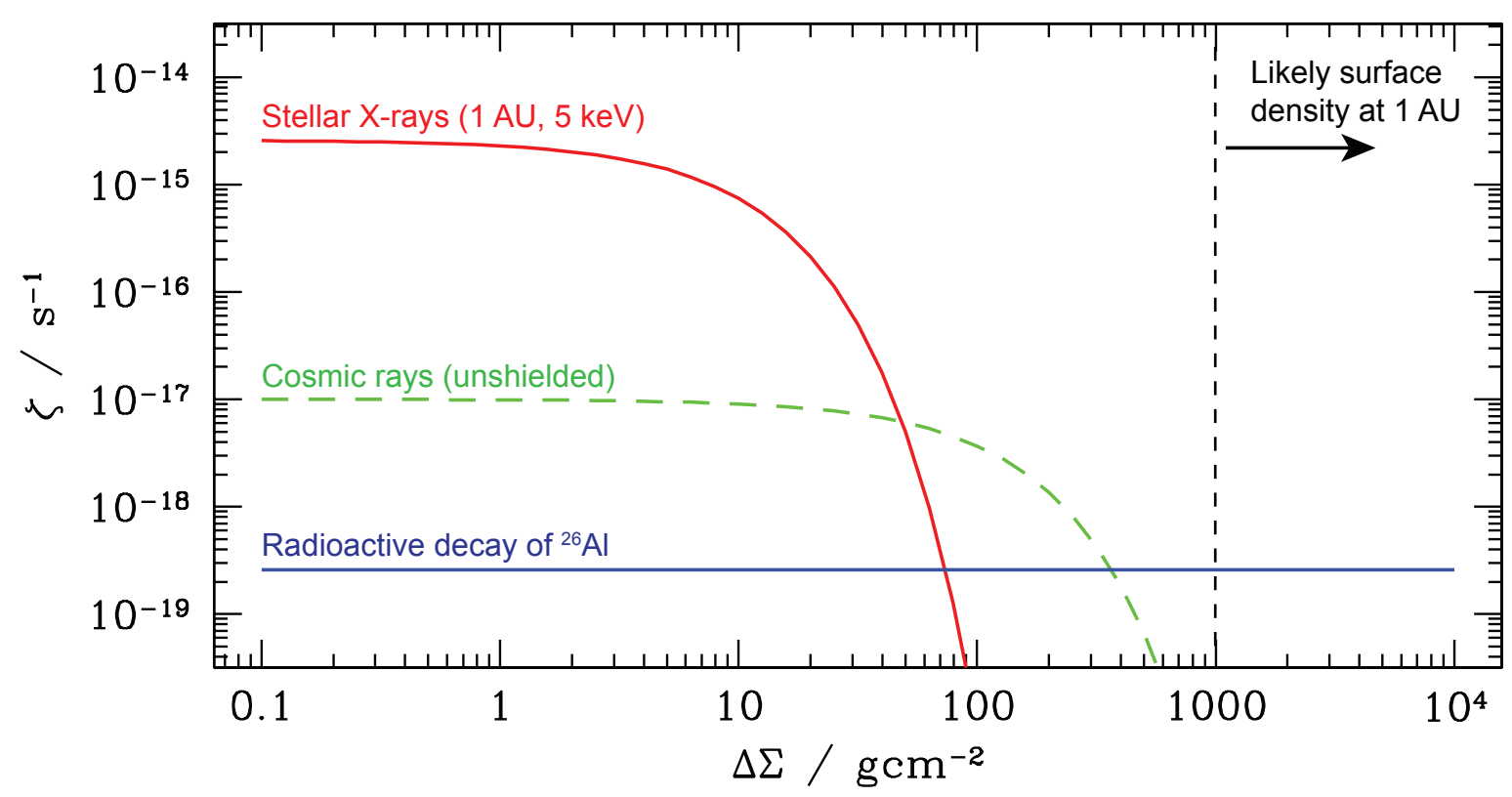

Figure 6: The dependence of the ionization rate $\zeta$ on the column density $\Delta \Sigma$, measured from the disk surface. Contributions from the three three main sources of non-thermal ionization are shown: (a) stellar X-rays, evaluated at $1 \mathrm{AU}$ (as a function of radius, $\zeta \propto r^{-2}$ ) using the Turner \& Sano (2008) fit to the calculations of Igea \& Glassgold (1999), (b) cosmic rays, assuming that the flux is not attenuated by a stellar or disk wind, and (c) radioactive decay of the short-lived isotope ${ }^{26} \mathrm{Al}$ (Stepinski 1992). Although all three curves are approximate, they illustrate two key points. First, irradiation by stellar X-rays almost certainly dominates the ionization near the disk surface. Second, the likely column densities in the inner disk $\left(\Sigma \gtrsim 10^{3} \mathrm{~g} \mathrm{~cm}^{-2}\right)$ are sufficient to provide effective shielding of the mid-plane from incident X-rays or cosmic rays.

The simplest calculation of the recombination rate, due to Oppenheimer \& Dalgarno (1974), is based upon the equilibrium solution to a chemical network comprising ionization together with generic versions of the three reactions listed above. This network is simple enough that it can be solved analytically in some limits (e.g. Equation 24, if metal ions can be neglected), but it is already instructive. In particular, it reveals that the ionization fraction is sensitive to trace amounts of metal atoms. Their abundance is important because molecular ions (which would otherwise recombine efficiently with electrons), tend to transfer charge to the metals which then recombine relatively slowly (Fromang, Terquem \& Balbus 2002). This physical effect is also present in more refined calculations, which model reactions between large numbers of chemical species (Bai \& Goodman 2009 Ilgner \& Nelson 2006; Sano et al. 2000; Semenov, Wiebe \& Henning 2004), though at a weaker level, because the more complex models include additional avenues for recombination of molecular ions. The issue is discussed in detail by Ilgner \& Nelson (2006), who present a detailed comparison of the ionization fraction predicted by different reaction networks.

Small dust grains, if they are present in any significant abundance, generally result in faster recombination. The collision rates between grains and charged particles have been computed by Draine \& Sutin (1987), who give expressions for recombination rates that include the effects of charged grains, and the induced polarization of neutral grains by electrons or ions (a clear discussion in the disk context is given by Bai \& Goodman 2009). These subtleties are important, but 
even a crude estimate suffices to demonstrate that small grains can radically alter the gas-phase recombination rate. Let us consider a disk with gas density $\rho$ and dust-to-gas ratio $f_{\mathrm{d}}$, in which the dust particles all have radius $a$ and material density $\rho_{\mathrm{d}}$. The surface area per unit volume presented by the dust particles is $S=3 f_{\mathrm{d}}\left(\rho / \rho_{\mathrm{d}}\right) a^{-1}$, and the geometric estimate of the recombination rate due to collisions with molecular ions of number density $n_{I}$ and velocity $v_{I} \sim\left(k T / m_{I}\right)^{1 / 2}$ is $\sim S n_{I} v_{I}$. Comparing this to the rate of dissociative recombination, quoted in $\$ 3.2$, we find that the ratio of the rate of recombination on dust compared to its gas-phase value is,

$$
\frac{\dot{n}_{I, \text { dust }}}{\dot{n}_{I, \text { gas }}} \sim 20\left(\frac{f_{\mathrm{d}}}{10^{-2}}\right)\left(\frac{x}{10^{-12}}\right)^{-1}\left(\frac{T}{100 \mathrm{~K}}\right)\left(\frac{a}{1 \mu \mathrm{m}}\right)^{-1} .
$$

The conclusion, which is borne out by all detailed calculations, is that the ionization fraction in the non-thermal zone will be set largely by the abundance of grains (Bai \& Goodman 2009, Ilgner \& Nelson 2006, Sano et al. 2000). What matters most is the size of the smallest grains, since for a standard grain size distribution $\left(N(a) \propto a^{-3.5}\right.$, Mathis, Rumpl \& Nordsieck 1977) the largest contribution to the surface area comes from the smallest particles. The limiting case is a size distribution that contains a significant abundance of polycyclic aromatic hydrocarbons (PAHs). These grains have sizes of just $a \sim 0.01 \mu \mathrm{m}$ and hence are especially efficient at stimulating recombination (Bai \& Goodman 2009, Perez-Becker \& Chiang 2010, Sano et al. 2000).

The upshot of this discussion is that the ionization fraction within disks - outside of the thermally ionized inner region - is closely coupled to grain growth and grain settling. There is strong theoretical evidence that micron and sub-micron sized grains can coagulate rapidly, but this must be weighed against equally compelling observational evidence that at least some small particles survive throughout the disk lifetime (Dullemond \& Dominik 2005). Settling can also be rapid, but will be inhibited for small particles if there is any significant level of turbulence within the gas (Dubrulle, Morfill \& Sterzik 1995; Fromang \& Papaloizou 2006). Since the level of turbulence is likely to depend, in turn, on the ionization fraction, it is easy to speculate that the chain of dependencies might give rise to either self-limiting or cyclic behavior, in which the dust settles until the ionization fraction rises enough to ignite the MRI.

3.2.4 LAYERED ACCRETION AND DEAD ZONES An audacious synthesis of many of the ideas outlined above was attempted by Gammie (1996), who proposed that disks in which angular momentum transport occurred via the MRI ought to develop a "layered" structure. The idea, illustrated in a somewhat modified form in Figure 7) (with X-rays replacing cosmic rays as the main non-thermal ionization source, following Glassgold, Najita \& Igea 1997), is that the criteria for MRI activity define three radial zones,

1. An inner zone, where the disk is MRI-active and turbulent because the mid-plane temperature is high enough $\left(T \gtrsim 10^{3} \mathrm{~K}\right)$ to ionize the alkali metals. The outer boundary of this region, which is at about $1 \mathrm{AU}$ for $\dot{M}=10^{-7} M_{\odot} \mathrm{yr}^{-1}$ (Figure 2) will move inward as the disk accretion rate declines.

2. An outer zone, where non-thermal sources of ionization suffice to raise the ionization fraction at the mid-plane above the threshold for MRI activity. The inner boundary of this region will also move inward as the surface density drops, and the shielding the disk provides to ionizing radiation and particles becomes less effective.

3. An intermediate region, where the mid-plane is cool enough, and well-enough shielded from ionizing radiation, to fail to satisfy the conditions for the MRI to operate. Gammie (1996) suggested that the disk at these radii would develop a layered structure, with a "dead zone" near the mid-plane in which turbulence was absent or strongly suppressed. Accretion would then occur entirely (or primarily) through an active surface layer, whose thickness is defined by the flux and penetration strength of cosmic rays (in the original version) or stellar X-rays. 


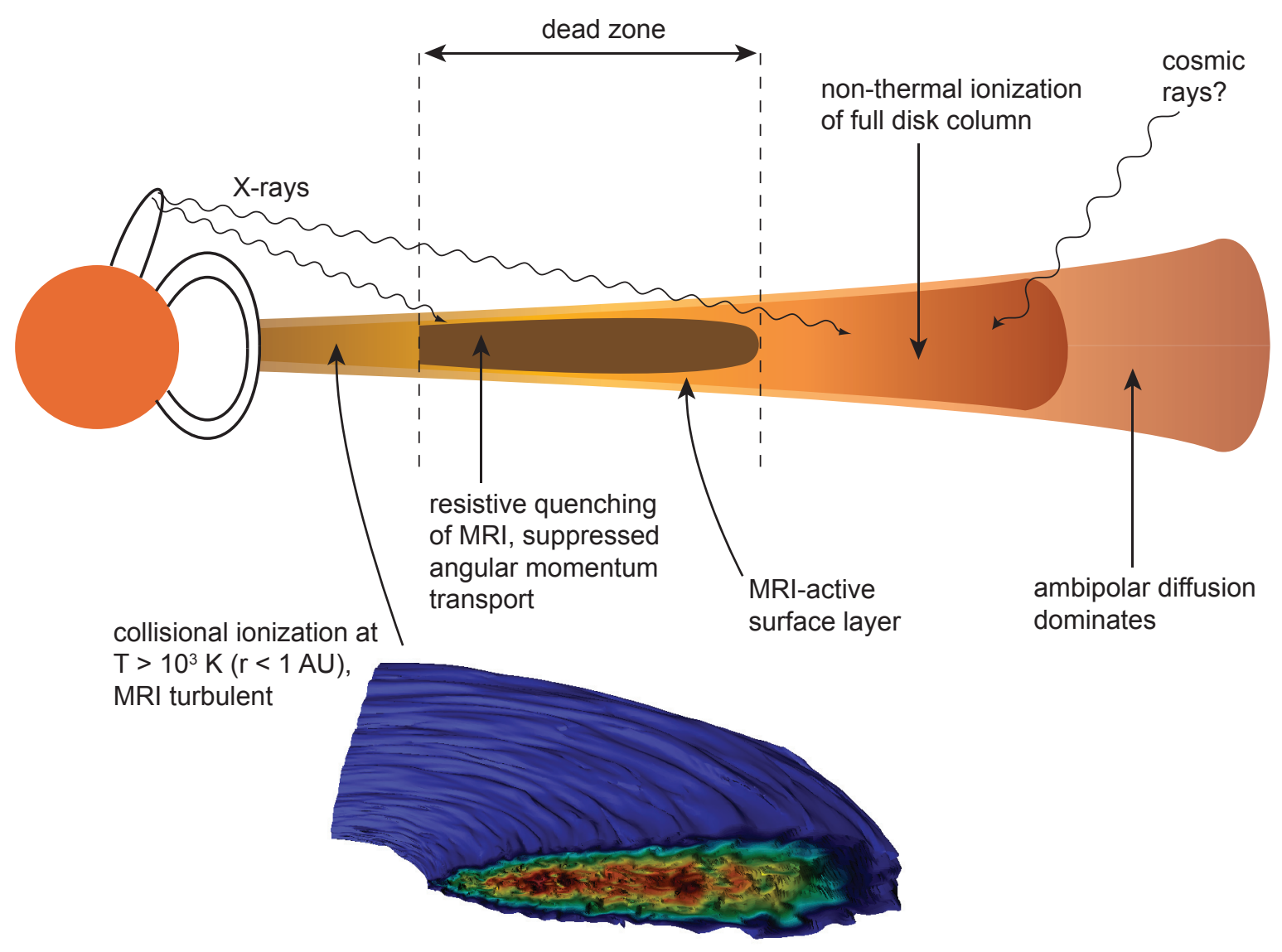

Figure 7: Schematic structure of the protoplanetary disk if the low ionization fraction at radii $r \sim 1$ AU quenches angular momentum transport due to the MRI, forming a "dead zone" (Gammie 1996). X-rays, produced from the cooling of plasma confined within magnetic field loops in the stellar corona, ionize the disk surface, but fail to penetrate to the mid-plane. The inset figure shows density isosurfaces computed from a simulation of a fully turbulent disk (Beckwith, Armitage \& Simon 2011).

No observation provides direct evidence either for or against the existence of dead zones. Theoretical calculations, however, continue to suggest that it is more likely than not that protoplanetary disks develop a dead zone at radii $r \sim 1$ AU (Bai \& Goodman 2009; Salmeron \& Wardle 2008; Terquem 2008; Turner, Carballido \& Sano 2010; Turner \& Drake 2009). The sole situation in which a region of suppressed MRI transport is not predicted to exist is the case where there is a strong depletion of small grains. It is worth noting that, although all of these authors hew to the same basic considerations of non-ideal MHD physics, there are many significant differences between the technical approaches that they adopt. Salmeron \& Wardle (2008), for example, assume a minimummass model for the disk surface density profile (Hayashi 1981), within which they compute the detailed vertical structure of linear MRI modes (see also Salmeron \& Wardle 2005). Terquem (2008), on the other hand, adopts a simpler model for the MRI physics but solves self-consistently (in the context of a one-dimensional vertically averaged disk model) for the steady-state surface density distribution of the disk. Although the problem is undoubtedly messy, the general agreement between these calculations lends weight to the contention that some sort of dead zone does exist.

The existence of dead zones, falling as they do squarely in the midst of the radii of greatest interest for terrestrial planet formation, has multiple broader ramifications. The most important is 
probably the effect that a dead zone would have on planetesimal formation, since many candidate mechanisms for building planetesimals are sensitive to the level of intrinsic disk turbulence (Chiang \& Youdin 2010). Generically, a dead zone also results in an enhanced surface density across the radii where the MRI is suppressed, and can - if the suppression is complete enough - admit new mechanisms for time-dependent disk outbursts (Armitage, Livio \& Pringle 2001; Gammie 1999; Zhu et al. 2010).

Going beyond the basic question of whether dead zones exist, it is important to know both the strength and the nature of any residual turbulence and angular momentum transport that persists in the MRI-inactive layer. Although the original models for layered accretion typically assumed entirely laminar, torque-free dead zones, subsequent work has identified three processes that likely animate the mid-plane to some degree. First, hydrodynamic waves, excited by the turbulence in the active surface layers, can penetrate to the mid-plane and exert a non-zero Reynolds stress on the magnetically inert gas there (Fleming \& Stone 2003, Oishi \& Mac Low 2009). In the vertically stratified local simulations of Fleming \& Stone (2003), the mid-plane Reynolds stress in the dead zone was found to be surprisingly large - less than an order of magnitude below the Maxwell stress in an MRI-active disk. Those simulations used an isothermal equation of state, and the true strength of Reynolds stresses communicated to the mid-plane may well be sensitive both to that assumption (Bai \& Goodman 2009) and to the thickness of the active surface layer. Nonetheless, a non-zero dead zone stress due to this effect seems very probable. (Direct activation of the dead zone, due to turbulent mixing of ionized gas downward before it has time to recombine, has also been suggested, and can be effective in the absence of dust; Ilgner \& Nelson 2008; Turner, Sano \& Dziourkevitch 2007). Second, radial magnetic fields can diffuse to the mid-plane, where shear suffices to generate an azimuthal component (Turner \& Sano 2008). The combination of radial and azimuthal fields then yields a laminar Maxwell stress $\left\langle B_{r} B_{\phi}\right\rangle$, which can transport angular momentum (Equation 6) without, in principle, being accompanied by turbulent motions. This physics is related to that driving the time-dependent behavior observed by Simon, Hawley \& Beckwith (2010) in moderately resistive disks (where the azimuthal field eventually grows strong enough to reignite the MRI even in the mid-plane), and leads one to suspect that the radial edges of dead zones may be significant wellsprings for accretion variability. Finally, Latter, Bonart \& Balbus (2010) have identified a linear buoyancy-type instability that may operate in dead zones where resistive diffusion is much faster than thermal diffusion. This instability illustrates the general point that turbulence is not synonymous with angular momentum transport, since it would likely result in turbulence and mixing without significant attendant transport.

Thus far, work on MHD processes within protoplanetary disks has focused on dynamics. Crudely put, what is the value of $\alpha$, and how can that be related to observations of disk evolution? This emphasis should not blind us to the fact that other connections between observations and theory may ultimately be more powerful. King \& Pringle (2010), for example, have emphasized that the energetic requirements for forming chondrules (for a review, see Scott 2007) are substantial, and that meteoritic constraints from the Solar System can therefore inform models of accretion (whether by the MRI, gravitational instability, or some other process). A limited amount of work has already been done in this direction. Joung, Mac Low \& Ebel (2004) suggested that the temperatures attained in current sheets could reach values high enough to form chondrules late in the disk evolution, when dust had settled and ambipolar diffusion was important (the role of ambipolar diffusion in modifying the small-scale structure of MHD turbulence is discussed by Brandenburg \& Zweibel 1994). Additional work along these lines, including simulations in the Hall / Ohmic regime that is expected to prevail on AU scales at earlier times during disk evolution, would be valuable. 


\subsection{Baroclinic instability and vortices}

The existence of dead zones in protoplanetary disks means that we have to worry about the effects of possible hydrodynamic instabilities, even if their angular momentum transport efficiency is low. In this sense protoplanetary disks are quite distinct from other astrophysical disk systems, in which purely hydrodynamic instabilities, even if they theoretically exist, would likely be swamped by the MRI. At the outset, it is useful to distinguish between instabilities that owe their origin purely to the shear (in the MHD case, the MRI is an example of such an instability), and those which instead feed off an unstable entropy gradient. For shear-driven instabilities, theoretical arguments (e.g Lesur \& Longaretti 2005) and, to a limited extent, laboratory experiments (Ji et al. 2006), have largely excluded the existence of instabilities that would yield efficient angular momentum transport. Purely hydrodynamic disks are linearly stable, and, although perturbations within such disks can exhibit transient growth (Afshordi, Mukhopadhyay \& Narayan 2005, Chagelishvili et al. 2003, Ioannou \& Kakouris 2001; Rebusco et al. 2009), no avenue that leads to fully-fledged turbulence has been demonstrated.

Entropy-driven instabilities are more promising. Klahr \& Bodenheimer (2003) observed an instability, which led to vortex formation and angular momentum transport, in global numerical simulations of disks with an outwardly declining entropy profile. These early simulations, however, were not able to clearly characterize the nature of the instability (for example, whether it was local or global), or the physical prerequisites for its existence. Subsequently, Petersen, Julien \& Stewart (2007a b), using two-dimensional global simulations of unmagnetized disks, and Lesur \& Papaloizou (2010), using local simulations, confirmed that a disk with a linearly stable radial entropy gradient could nevertheless be unstable to the baroclinic generation of vorticity. The instability is known as the "subcritical baroclinic instability" (SBI).

Compared to the linear instabilities that anchor discussions of the MRI and disk self-gravity, the SBI is altogether more subtle. As currently understood, it is a non-linear (or "subcritical") instability that relies for its existence on the interplay of two distinct thermodynamic properties of the disk, (1) a radially unstable entropy gradient and, (2) radiative cooling (or thermal diffusion) at a rate that promotes the baroclinic generation of vorticity (Lesur \& Papaloizou 2010; Petersen, Julien \& Stewart 2007a). The first of these properties is related to the stability of a rotating flow to convection, and can readily be quantified. The importance of buoyancy (in $r$ ) is expressed via the Brunt-Väisälä frequency,

$$
N_{r}^{2}=-\frac{1}{\gamma \rho} \frac{\mathrm{d} P}{\mathrm{~d} r} \frac{\mathrm{d}}{\mathrm{d} r} \ln \left(\frac{P}{\rho^{\gamma}}\right)
$$

where $P=P(r)$ and $\rho=\rho(r)$ are the pressure and density profiles of the disk and $\gamma$ is the adiabatic index. In the absence of shear, the flow is unstable to the linear growth of perturbations if,

$$
N_{r}^{2}<0
$$

which is the Schwarzschild criterion for the onset of convection. Including shear, the condition for linear instability to axisymmetric perturbations becomes the more stringent Solberg-Hoïland criterion, which can be written for a Keplerian disk as,

$$
N_{r}^{2}+\Omega_{\mathrm{K}}^{2}<0
$$

For the SBI to operate; the background structure of the disk must be Schwarzschild unstable (but Solberg-Hoïland stable, thereby avoiding linear instability) in the radial direction.

Petersen, Julien \& Stewart (2007a) and Lesur \& Papaloizou (2010) have studied the evolution of a Schwarzschild unstable disk that is initially seeded with finite amplitude temperature or vorticity perturbations. Vorticity is not a conserved quantity in such a setup, because there is a non-zero baroclinic term $\nabla P \times \nabla \rho$ that acts a source or sink of vorticity. Irrespective of the thermal physics 


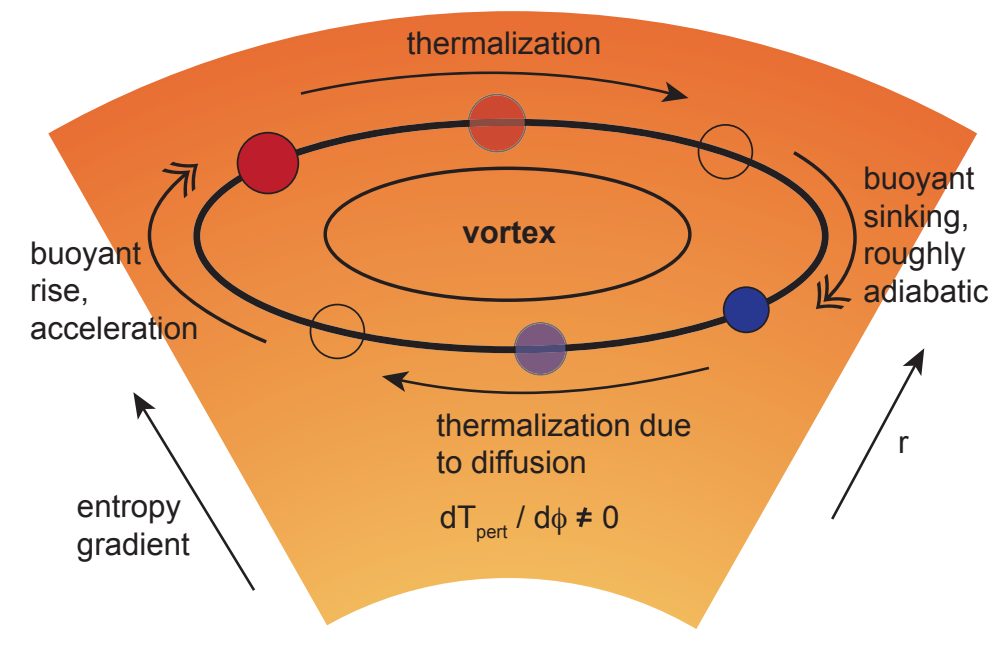

Figure 8: Schematic illustration (after Lesur \& Papaloizou 2010; Petersen, Julien \& Stewart 2007a) showing how the baroclinic instability sustains non-linear vortices in a disk with a radial entropy profile. Fluid parcels moving either inward or outward are subject to buoyancy forces, and arrive at their new radial location either hotter or cooler than the background disk. As they drift azimuthally, diffusive processes equilibrate their temperature until it matches the background. As a consequence, the vortex sets up a temperature perturbation $\mathrm{d} T_{\text {pert }} / \mathrm{d} \phi \neq 0$, maintaining the baroclinic term in the vorticity equation that drives the vortex.

of the disk, the flow will initially develop an organized pattern of vortices. In the absence of radiative cooling and heating, or of thermal diffusion, however, numerical simulations show that the vortices eventually decay. A self-sustaining, non-linear instability - the SBI - is only present when thermal or radiative diffusion occurs on an adroitly chosen time scale, neither too fast, which would reduce the effects of buoyancy, nor too slow, which would make the flow around the vortex almost adiabatic. Under the appropriate conditions, it is possible to set up a cycle, illustrated in Figure 8 , in which dissipative processes are balanced by work done by buoyancy as fluid circulates around a self-sustaining vortex.

Although the existence of the SBI in model systems is now securely established, we have only paltry knowledge the outcome of the instability, especially in three dimensions, where vertical stratification is sure to introduce new effects (Barranco \& Marcus 2005). The simulations of Lesur \& Papaloizou (2010) find that the instability is strongest for a diffusion time scale that is of the order of $10 \Omega_{\mathrm{K}}^{-1}$, and suggest that density waves generated by the vortices (observed in compressible simulations) may generate a weak outward flux of angular momentum (Johnson \& Gammie 2005). It is also unclear under what physical conditions the SBI could play an important role in protoplanetary disks. For a disk to be Schwarzschild unstable, we require some combination of a relatively steep radial temperature profile, and a relatively shallow density profile. Specifically, if the mid-plane density and temperature scale as $\rho \propto r^{-\beta_{\rho}}$ and $T_{c} \propto r^{-\beta_{T}}$, respectively, then entropy declines outward if (Klahr \& Bodenheimer 2003),

$$
\beta_{T}-(\gamma-1) \beta_{\rho}>0
$$

The temperature gradient at the mid-plane is likely to be steeper in disks whose thermal structure is dominated by viscous dissipation, rather than by external irradiation, and taken in isolation this suggests that the SBI might be most important in dense disks at early times, when accretional 
heating can in principle overwhelm irradiation. The requirement for a relatively short radiative cooling time scale, however, works in the opposite direction, since short cooling times occur for low mass disks of modest optical depth (e.g. Figure 2).

As with the MRI and self-gravity, interest in the SBI and disk vortices is not solely - or in this case even primarily - motivated by dynamical considerations. Vortices concentrate solid particles toward their cores (Barge \& Sommeria 1995, Chavanis 2000, Tanga et al. 1996), which as a result become sites of locally enhanced solid-to-gas ratio. The existence of vortices could therefore have profound implications for planetesimal formation, particularly if planetesimals form via a mechanism that requires a threshold solid-to-gas ratio to be exceeded (an example being the streaming instability; Johansen, Youdin \& Mac Low 2009).

\subsection{Convection}

Convection has probably generated more vigorous instability among theorists than it does in typical protoplanetary disks. Early disk models, notably that of Lin \& Papaloizou (1980), were based upon the assumption that convective cells, with characteristic velocity $v_{c}$ and scale $l \sim v_{c} / \Omega_{\mathrm{K}}$, would transport angular momentum with an effective kinematic viscosity $\nu=v_{c}^{2} / \Omega_{\mathrm{K}}$. Standard mixing length theory was used to calculate $v_{c}$ as a function of the vertical disk structure. These models were later abandoned in the face of persuasive numerical and analytic evidence suggesting that vertical disk convection was not merely an inefficient source of viscosity, but actually worked to transport angular momentum in the wrong direction, inward! Very recently, however, sentiment has reversed sign again. Lesur \& Ogilvie (2010) have completed high resolution incompressible simulations of disk convection that show outward transport of angular momentum, with efficiencies that under reasonable disk conditions might yield $\alpha \sim 10^{-4}$ or even larger. The contrary results of previous investigators can be traced to numerical shortcomings (early simulations were not able to access the strongly turbulent regime of convection that ought to prevail in real disks), and to the use of analytic approximations that prove to be invalid.

The new results are important as a demonstration of how a fundamental physical process operates in a disk geometry, but it is as yet unclear whether they presage a revival of disk models in which convection plays a leading role. Convection cannot be important in an externally irradiated disk, and the current idealized simulations do not model the viscous dissipation that, in a self-consistent theoretical description, would have to sustain the unstable stratification. Moreover, although Lesur \& Ogilvie (2010) obtain outward transport, they also confirm the intuitive expectation that convective turbulence is more adept at moving heat (vertically) than it is at moving angular momentum (radially). This observation certainly limits the efficiency of a convectively-driven disk, but, until more work has been done to confirm the new results and establish the fundamental properties of disk convection, more definitive statements are speculative.

\subsection{Planet-driven disk evolution}

Theoretical estimates of the time needed to assemble the core of a gas giant planet (Movshovitz et al. 2010) are, to within uncertain factors of a few, equal to the observed lifetime of protoplanetary gas disks. Several authors have speculated that this apparent coincidence might, to the contrary, signal a causal relationship; perhaps the formation of planets itself catalyzes angular momentum transport and results in the viscous dispersal of the gas disk (Goodman \& Rafikov 2001, Sari \& Goldreich 2004). The physical process that can mediate such an unexpected coupling is well known. Gravitational torques between a planet and a gas disk, exerted at radial locations within the disk that correspond to Lindblad resonances, transfer angular momentum outward in a manner qualitatively resembling a viscous process (Goldreich \& Tremaine 1980).

In its simplest version, the idea that planets generate the bulk of angular momentum transport 
within disks almost certainly fails. Although, in principle, a low-mass planet embedded within the gas disk could passively shuttle angular momentum between the disk at its inner and outer Lindblad resonances, while remaining on a fixed orbit, no calculation suggests that gravitational torques operate in this fashion. Rather, calculations show that the net torque on the planet (due to the sum of Lindblad and corotation torques) is a significant fraction of the sum of the absolute values of the torques (this is the well known problem of Type I migration, e.g. Lubow \& Ida 2010). As a result, a population of low mass planets would only be able to catalyze the accretion of a relatively small mass of gas - of the order of the total mass in planets - before either spiraling into the star or away to large disk radii.

Nonetheless planets - especially massive gas giants - may still play a significant if more limited role in disk evolution. The interpretation of transition disk sources, observational aspects of which are reviewed by Williams \& Cieza (2011) in this volume, remains unclear, but it is undisputed that the existence of large inner holes in transition disks cannot be explained by simple viscous processes acting within the gas. A single massive planet, which opens a gap in the gas disk via the action of gravitational torques while allowing some gas to flow past the planet toward the star, could account for the observed properties of some transition disk sources (Calvet et al. 2002, Lubow \& D'Angelo 2006, Rice et al. 2003b). In other instances, however, the overlapping gravitational torques from multiple gas giant planets (Morbidelli \& Crida 2007, Z. Zhu, private communication), acting in concert more like a viscous process, may be needed if one is to explain the data within a planetary scenario.

\subsection{Section summary}

Determining the strength and nature of angular momentum transport is particularly difficult for protoplanetary disks, since no single physical mechanism is clearly dominant. A robust theoretical determination of $\alpha(r, z, t)$ is not possible, but enough is known about candidate transport mechanisms to make predictions that could be tested with future disk observations. To summarize this Section:

- Disk self-gravity (at early times), and the magnetorotational instability, are likely the most important dynamical agents in viscous protoplanetary disks. Other processes can also transport angular momentum, but have less general applicability.

- The efficiency of the MRI is a partially-known function of the magnitude of non-ideal MHD terms. In a real disk, these will vary with the stellar properties (since X-ray ionization is an important process), and with the extent of dust coagulation (since small dust particles largely determine the recombination rate). Models that include the non-ideal physics are uncertain, but most of them predict the existence of a dead zone, with reduced levels of turbulence, at radii of the order of an $\mathrm{AU}$.

- The possibility that magnetic braking dominates angular momentum loss from some disks must be kept in mind. Even if it does not, weak large-scale magnetic fields threading the disk affect the efficiency of MRI-driven transport.

\section{DIFFUSION AND MIXING}

Thus far, we have proceeded under the assumption that angular momentum transport within the disk derives from a turbulent process. Turbulence is also, generically, efficient at mixing different fluids together, so if our working assumption is valid there ought to be a close connection between angular momentum transport, disk evolution, and the diffusion of trace gas species and dust through the disk. To quantify that connection, we assume that the disk contains some dynamically 
unimportant trace species of gas, with density $\bar{\rho}$, that mixes vertically according to a standard diffusion equation,

$$
\frac{\partial \bar{\rho}}{\partial t}=\frac{\partial}{\partial z}\left[D \rho \frac{\partial}{\partial z}\left(\frac{\bar{\rho}}{\rho}\right)\right]
$$

where $D$, the vertical gas diffusion coefficient, has the same units as viscosity $\left(\mathrm{cm}^{2} \mathrm{~s}^{-1}\right)$. The relative efficiency with which the turbulence transports angular momentum, and mixes gas vertically, can then be measured via the Schmidt number,

$$
\mathrm{Sc}=\frac{\nu}{D}
$$

A Schmidt number Sc $>1$ thus means that the turbulence is relatively poor at mixing for a given angular momentum transport efficiency. Very similar considerations apply to radial diffusion, although for the radial case the concentration $\bar{\rho} / \rho$ must be obtained from the solution to an advection-diffusion equation that accounts for the radial flow of the background disk (Clarke \& Pringle 1988).

The vertical Schmidt number has been measured for trace gas species (and for small dust particles, which when tightly coupled to the gas by aerodynamic forces behave similarly) using both local and global MRI simulations (Carballido, Stone \& Pringle 2005; Fromang \& Nelson 2009; Ilgner \& Nelson 2008; Johansen \& Klahr 2005; Turner et al. 2006). The dimensional argument that $D \sim \nu$ turns out to be reasonable; typical values of the vertical Schmidt number extracted from simulations are $\mathrm{Sc}=1-3$. Significant vertical variations occur - unsurprisingly since the properties of fluid turbulence driven by the MRI vary markedly with height above the mid-plane - and must be included in order to model the vertical distribution of diffusing species accurately (Fromang \& Nelson 2009). Substantially higher Schmidt numbers result if the turbulence within the disk is stimulated by the presence of net vertical fields (Johansen, Klahr \& Mee 2006).

Larger particles, that are imperfectly coupled aerodynamically to the turbulent motions within the gas, are expected to diffuse less readily than gaseous contaminants (Cuzzi, Dobrovolskis \& Champney 1993, Youdin \& Lithwick 2007). The ratio of the particle diffusion coefficient, $D_{p}$, to that of the gas, $D$, can be estimated analytically using a model for the structure of the turbulence within the disk. It depends upon the strength of the coupling between the particles and the turbulent gas, measured via the dimensionless stopping time,

$$
\tau_{s}=\Omega_{\mathrm{K}} t_{s}
$$

where $t_{s}$ is the exponential decay time for damping of relative motion between the particle and the gas. With this definition, Youdin \& Lithwick (2007) obtain,

$$
\frac{D_{p}}{D} \sim\left(1+\tau_{s}^{2}\right)^{-1}
$$

The diffusion of particles with dimensionless stopping times exceeding unity is thus predicted to be severely curtailed. Although variation of $\nu / D_{p}$ with stopping time (in the expected sense) has been seen in studies of particle diffusion within MRI turbulent disks (Fromang \& Nelson 2009), an accurate test of this scaling (which differs from previously widely used results) is not yet available.

\section{PHOTOEVAPORATION}

The observation of accretion signatures, generated when infalling gas strikes the stellar photosphere (Calvet \& Gullbring 1998), demonstrates that angular momentum transport or loss processes in the disk do occur on astronomically relevant time scales. This does not mean, however, that angular momentum transport processes are necessarily the primary agents for protoplanetary disk 
evolution. Mass loss from the disk is also likely to occur, perhaps especially at moderate to large distances from the star where the gravitational potential well is not too deep. If the mass loss rate is significant enough - roughly speaking if it exceeds the accretion rate $\dot{M}$ - then it will be the physics of mass loss rather than that of angular momentum transport that largely determines properties of the disk such as the surface density profile. This possibility needs to be kept in mind when interpreting observations of disks on large scales, where the local time scale for mass loss is plausibly shorter than that for angular momentum transport.

Here, we review work on disk mass loss driven by photoevaporation (Bally \& Scoville 1982). The importance of photoevaporation depends upon the strength and spectrum of radiation incident on the disk - either from the central star or from neighboring stars, if they are luminous enough as to dominate the flux of energetic photons - and upon the composition of the disk's upper layers. Although estimates of the resultant mass loss rate are uncertain, photoevaporation is very likely an important process for many disks, and may dominate the evolution in the Classical T Tauri phase for low mass stars in rich clusters, and for isolated stars that are particularly strong X-ray sources.

5.0.1 Essential PHYSiCs The essential physics of photoevaporation is illustrated in Figure 9 (after Shu, Johnstone \& Hollenbach 1993). Ionizing or dissociating radiation from the central star, or from external stars, impinges on the upper and lower surfaces of the disk and heats a relatively thin skin of gas there to a temperature $T_{\text {surf }}>T_{\text {eff }}$. At some radius $r_{g}$, the sound speed $c_{s}$ in the surface layer equals the local Keplerian velocity, where $r_{g}$ is given by,

$$
r_{g}=\frac{G M_{*}}{c_{s}^{2}}
$$

At radii $r \gtrsim r_{g}$ the gas in the surface layer is unbound (even neglecting the effects of rotation), and there is no impediment to it flowing freely away from the disk as a wind driven by thermal pressure gradients. For gas with mean molecular weight $\mu$, that has a number density at the base of the heated layer $n$, dimensional arguments suggest that the local mass loss rate ought to be,

$$
\dot{\Sigma}_{\text {wind }}(r) \sim \mu n(r) c_{s}(r) .
$$

Magnetic fields may be present, but need play no role at all in the driving of the wind. If they can be ignored, then the specific angular momentum of gas in the wind matches that at the surface of the disk where each outflowing streamline originates. In its simplest form, then, photoevaporation is a "pure" mass loss process, that diminishes $\Sigma$ locally at each radius without causing additional evolution due to angular momentum loss.

The radial dependence of the disk mass loss rate is determined by the interplay of radiation physics (the spectrum of the irradiating sources, and radiative transfer effects that alter the distribution of the flux seen by the disk), thermal physics in the disk (which will determine $T_{\text {surf }}$ ), and hydrodynamics (which finally determines $\dot{\Sigma}_{\text {wind }}$ ). Photoevaporation models can be divided into two classes depending upon the origin of the disk-irradiating flux. "External" photoevaporation models apply when the radiation that drives mass loss originates from other stars, usually massive stars in young clusters that have prodigious ultraviolet luminosities (Adams et al. 2004; Johnstone, Hollenbach \& Bally 1998). On average, the importance of external photoevaporation increases with the mass and compactness of the stellar cluster within which a disk finds itself (Armitage 2000, Fatuzzo \& Adams 2008). For disks around stars forming in groups or small clusters, such as Taurus, mass loss must instead be driven internally, by radiation from the central star. (Even in relatively rich clusters, internal photoevaporation can be the most important process at $r \sim \mathrm{AU}$, due to the $1 / r^{2}$ dependence of the central stellar flux.)

5.0.2 X-RAY And UV-Driven photoevaporation At $100 \mathrm{AU}$, the simple argument given above (leading to Equation 44) would imply that the surface layer of the disk must be heated to $T \gtrsim 10^{3} \mathrm{~K}$ in order to initiate a photoevaporative flow. This is in fact unduly pessimistic; more 


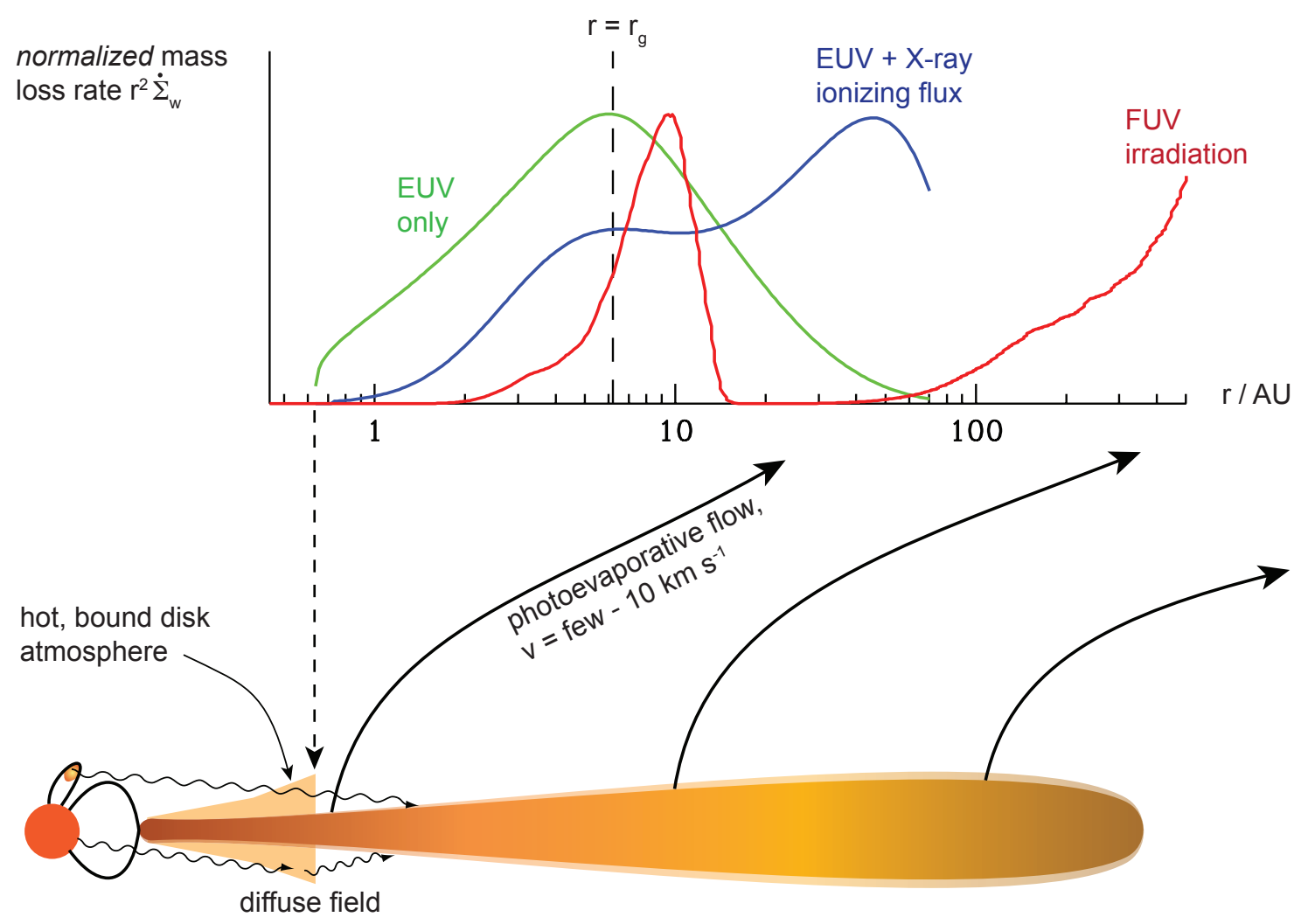

Figure 9: Mass loss by photoevaporation occurs when FUV, EUV or X-ray radiation heats the disk surface to a temperature such that the gas is unbound. The normalized mass loss rate $r^{2} \dot{\Sigma}_{w}$ is shown as a function of radius for three models: (1) EUV only, using an analytic fit to simulation results by Font et al. (2004) for $M_{*}=0.7 M_{\odot},(2)$ an EUV plus X-ray model (Owen et al. 2010), also for $M_{*}=0.7 M_{\odot}$, and (3) an FUV model for $M_{*}=M_{\odot}$ (using data smoothed from Figure 5 of Gorti, Dullemond \& Hollenbach 2009). The EUV component drives mass loss primarily near a characteristic radius $r \sim r_{g}=G M_{*} / c_{s}^{2}$ (where $c_{s}$ is the sound speed in the ionized gas), while $\mathrm{X}$-rays, produced in a magnetized stellar corona, or FUV irradiation produce a component of mass loss weighted to larger radii. The absolute mass loss rates depend upon the stellar spectrum. Note that different assumptions and computational methods were used to produce each of the three mass loss curves, which are therefore illustrative rather than strictly comparable.

detailed hydrodynamic models show that gas can escape at a reduced rate from radii as small as $r \simeq 0.1 r_{g}$ (Begelman, McKee \& Shields 1983; Font et al. 2004; Liffman 2003). Even with this boost, however, photoevaporation requires surface temperatures high enough that they can only be attained as a result of disk irradiation by energetic photons,

1. Far-ultraviolet (FUV) radiation, with $6 \mathrm{eV}<h \nu<13.6 \mathrm{eV}$, that is able to dissociate hydrogen molecules but not ionize hydrogen atoms.

2. Extreme-ultraviolet (EUV) radiation, with $13.6 \mathrm{eV}<h \nu<100 \mathrm{eV}$, capable of ionizing hydrogen.

3. X-rays, defined by convention as photons with $h \nu>0.1 \mathrm{keV}$.

The rate of mass loss depends upon the original spectrum of the radiation, upon how much attenuation takes place along the line of sight to the disk surface, and upon how each type of radiation 
interacts and heats the disk surface.

The central region of the Orion nebula provides a good environment in which to test external photoevaporation models, since both the relevant irradiating flux (FUV and EUV radiation from massive stars, especially the $\mathrm{O} 6 \operatorname{star} \theta^{1}$ Ori C), and the structure of the resulting photoevaporative flows, can be reliably inferred or directly observed (O'Dell, Wen \& Hu 1993). The Orion proplyd LV 2, for example, which is seen in projection to lie within the bright stars of the Trapezium, has a measured photoevaporative mass loss rate of $\dot{M}_{\text {wind }} \simeq 6 \times 10^{-7} M_{\odot} \mathrm{yr}^{-1}$, with an estimated error of only $\sim 10 \%$ (Henney et al. 2002, Vasconcelos et al. 2005). The observations in Orion are generally found to be consistent with theoretical models for external photoevaporation (Adams et al. 2004, Clarke 2007; Johnstone, Hollenbach \& Bally 1998; Richling \& Yorke 2000; Störzer \& Hollenbach 1999), which show that such high rates of mass loss occur when large $\left(\sim 10^{2}\right.$ AU) and massive disks are placed in close proximity to massive stars. The dominant driver of photoevaporation under these conditions is FUV radiation, which heats the disk surface to a temperature that ranges between a few $\times 10^{2} \mathrm{~K}$ (for a modest flux of incident FUV, specified as $G_{0}=300$, where $G_{0}=1$ corresponds to a flux of $1.6 \times 10^{-3} \mathrm{erg} \mathrm{cm}^{-2} \mathrm{~s}^{-1}$ between $91.2 \mathrm{~nm}$ and $200 \mathrm{~nm}$ ), up to $10^{3} \mathrm{~K}$ for $G_{0}=3 \times 10^{4}$ (Adams et al. 2004). For disks that are large enough, $r \geq 0.1-0.2 r_{g}$, the FUVdriven flow is dense enough as to completely absorb EUV radiation, whose strength and effects are therefore immaterial. Over time, the outer radius of the disk shrinks and the mass loss rate drops, precipitously once $r_{\text {out }} \ll r_{g}$. The main effect of external photoevaporation is thus not to destroy disks entirely (though it does curtail their lifetime), but rather to truncate their sizes. For sufficiently strong FUV fluxes $\left(G_{0} \gtrsim 10^{4}\right)$ the truncation radius of $0.1-0.2 r_{g}$ corresponds to radii of 10-20 AU, which is small enough to significantly limit the formation of gas giant planets in rich clusters.

Internal photoevaporation models are more complex, because a disk around a low mass star may be significantly influenced by radiation in all three bands: FUV, EUV, and X-ray. The flux seen by the disk in these bands is moderately well determined. In the FUV, there is likely to be both a persistent chromospheric contribution, estimated by Gorti \& Hollenbach (2009) at $L_{\mathrm{FUV}} / L_{*} \approx 5 \times 10^{-4}$, and a larger component associated with accretion hot spots. An accretion rate of $\dot{M}=10^{-7} M_{\odot} \mathrm{yr}^{-1}$, for example, could yield an FUV luminosity of the order of $10^{32} \mathrm{erg} \mathrm{s}^{-1}$. X-ray luminosities $L_{X} \approx 2 \times 10^{30} \mathrm{erg} \mathrm{s}^{-1}$ (for Solar mass stars, Equation 30 ) are comparable to the chromospheric FUV values, though it should be noted that the dispersion in X-ray luminosity is large. EUV luminosities are more problematic, because absorption in the interstellar medium frustrates direct observation of the EUV flux from accreting, Classical T Tauri stars (CTTs). Estimates in this band are generally derived using scaling arguments (Kamp \& Sammar 2004), or indirect observational constraints (Alexander, Clarke \& Pringle 2005). For example, theoretical spectra, constructed assuming that the coronal properties of CTTs resemble those of RS CVn binaries (some of which lie near enough that their EUV is not completely absorbed), suggest that $L_{\mathrm{EUV}} \sim L_{X}($ Ercolano, Clarke \& Drake 2009). For reference, the kinetic luminosity of even a powerful wind - one with a mass loss rate of $10^{-8} M_{\odot} \mathrm{yr}^{-1}$ and a terminal velocity of $5 \mathrm{~km} \mathrm{~s}^{-1}$ - is only $L \approx 10^{29} \mathrm{erg} \mathrm{s}^{-1}$. Simple energetic arguments cannot therefore exclude any of the different radiation sources from playing a major role in disk mass loss. Observational evidence for photoevaporation driven by the central star is for now primarily indirect, being based, for example, on the comparison between observed [Ne II] 12.81 $\mu$ m line profiles (Herczeg et al. 2008, Pascucci \& Sterzik 2009) and theoretical models (Alexander 2008, Ercolano \& Owen 2010).

Historically, photoevaporation driven by an EUV flux from the star received the first detailed study. The initial motivation was to explain the formation of ultracompact HII regions as a result of photoevaporation of the disk of a massive star (Hollenbach et al. 1993, Yorke 1993), but it was shortly realized that similar processes could be significant for disks around low mass stars (Shu, Johnstone \& Hollenbach 1993). The physics in this case is greatly simplified by the fact that the temperature of photoionized gas is nearly a constant, $T_{\text {surf }} \approx 10^{4} \mathrm{~K}\left(c_{s} \approx 10 \mathrm{~km} \mathrm{~s}^{-1}\right)$, independent 
of uncertain aspects of the disk's chemical properties or of dust physics. Provided that the EUV is not absorbed close to the star (as may happen early on), the ionizing radiation produces a modest but securely determined rate of mass loss. For a disk that extends to close to the star (i.e. before any inner hole develops), the mass loss rate can be evaluated by adopting the analytic scaling of Hollenbach et al. (1994), scaled down by a factor of three as suggested by the numerical results of Font et al. (2004),

$$
\dot{M}_{\text {wind }} \approx 1.4 \times 10^{-10}\left(\frac{\Phi}{10^{41} \mathrm{~s}^{-1}}\right)^{1 / 2}\left(\frac{M_{*}}{M_{\odot}}\right)^{1 / 2} M_{\odot} \mathrm{yr}^{-1} .
$$

Here, $\Phi$, the stellar output of ionizing EUV photons, affects the mass loss rate only as the square root. This relatively weak scaling with $\Phi$, which is not qualitatively altered even if the wind contains dust (Richling \& Yorke 1997), ameliorates somewhat the large uncertainties attending its correct value. The radial distribution of the mass loss is plotted in Figure 9. EUV photoevaporation is most important across a relatively small range of radii, and peaks near $r_{g}$, at about 9 AU for a Solar mass star.

The relatively low rates of mass loss due to EUV photoevaporation can be attributed, in part, to the large cross section for absorption of ionizing photons by neutral gas. For the geometry shown in Figure 9 most EUV photons emitted by the star are absorbed by the tightly bound disk gas nearest the star, and the flux seen by the gas near $r_{g}$ is dominated by the diffuse field of photons produced as the inner gas recombines. Higher mass loss rates occur after the disk close to the star becomes optically thin to ionizing radiation, and the stellar flux can impinge directly upon the outer disk. In this "direct" regime of EUV photoevaporation, the mass loss rate is predicted to increase with the radius of the inner hole in the disk as $r_{\text {hole }}^{1 / 2}$ (Alexander, Clarke \& Pringle 2006a).

Recent work shows that the addition of the X-ray and / or FUV spectral components leads to much higher predicted mass loss rates (Gorti \& Hollenbach 2009, Owen et al. 2010). Both X-rays and FUV photons are more penetrating than EUV, and, although they do not heat the disk surface to as high a temperature as EUV, the boost in mass loss derived from launching the flow at a higher $n$ (Equation 45) has the potential to outweigh the lower $c_{s}$. These flows, however, are less forgiving of analytic approximations than is the case with EUV irradiation, which generates a sharply defined ionization front that serves as the launch point for the wind. For X-ray or FUV irradiation, it is necessary to solve numerically for both the hydrodynamic and thermal structure of the disk surface as it transitions into a wind. The thermal structure depends upon the irradiating spectrum, grain physics, chemical processes, and cooling by atomic and (where molecules are present) molecular lines (e.g. Gorti \& Hollenbach 2008; Woitke, Kamp \& Thi 2009). Accounting accurately for these effects remains challenging, with independent codes returning somewhat different predictions for the gas temperature even when the same physical assumptions are adopted (Röllig et al. 2007). As was the case for the ionization fraction $(\$ 3.2 .3)$ the abundance of PAHs and small grains is critical. If PAHs are present, Gorti \& Hollenbach (2008) find that their contribution to disk heating (via grain photoelectric emission) can be larger by a factor of $\sim 2$ than X-ray heating at $r \sim 10$ AU. Observationally, PAH spectral features are detected in only a small fraction of T Tauri stars (Geers et al. 2006, Oliveira et al. 2010). The non-detections could, however, be explained by relatively modest depletion of the surface PAH abundance (possibly as little as a factor of $\sim 10$; Geers et al. 2006), rather than by a complete absence of very small grains. This uncertainty in the PAH abundance (and in their properties) evidently propagates through to FUV photoevaporation models.

Owen et al. (2010) computed radiation hydrodynamic models of X-ray irradiated disks, assuming a luminosity $L_{X}=2 \times 10^{30} \mathrm{erg} \mathrm{s}^{-1}$. The input spectrum included an EUV component $\left(L_{\mathrm{EUV}}=L_{X}\right)$, while FUV irradiation was not considered. For a disk of mass $M_{\text {disk }}=0.026 M_{\odot}$, surrounding a $0.7 M_{\odot}$ star, they obtained a mass loss rate of $\dot{M}_{\text {wind }}=1.4 \times 10^{-8} M_{\odot} \mathrm{yr}^{-1}$, some two orders of magnitude greater than the EUV-only expectation. Absolute mass loss rates were locally higher 
than the EUV models at essentially all radii, but, when normalized to the total mass loss rate, the $\mathrm{X}$-ray wind was found to originate across a much broader range of disk radii, with the strongest contribution coming at large $(\approx 50 \mathrm{AU}$, see Figure 9 ) distances from the star. Even larger mass loss rates from the outer disk are likely in the presence of strong FUV irradiation. Gorti \& Hollenbach (2009) calculated the thermal and chemical structure of a disk exposed to the joint effects of FUV, EUV, and X-ray irradiation, from which they analytically estimated the likely mass loss rates. For an FUV luminosity of $L_{\mathrm{FUV}}=4 \times 10^{31} \mathrm{erg} \mathrm{s}^{-1}$ incident on a disk around a Solar-mass star, they estimated mass loss rates of $\sim 3 \times 10^{-8} M_{\odot} \mathrm{yr}^{-1}$, with much of the wind originating beyond 100 AU.

5.0.3 Disk Evolution Following the work of Clarke, Gendrin \& Sotomayor (2001), many authors have investigated how disks evolve under the combined influence of viscous angular momentum transport and photoevaporation (Adams et al. 2004 Alexander, Clarke \& Pringle 2006b; Gorti, Dullemond \& Hollenbach 2009; Matsuyama, Johnstone \& Hartmann 2003; Owen et al. 2010), and how such models may be constrained by observations of transition disk sources (Owen, Ercolano \& Clarke 2010) and by the statistics of extrasolar planets (Alexander \& Armitage 2009). The basic approach is to couple simple viscous disk models, that assume either a constant $\alpha$ or a fixed functional form for $\nu(r)$, with mass-loss profiles $\dot{\Sigma}(r)$ derived from analytic or hydrodynamic photoevaporation calculations. The wind is assumed to carry away the same specific angular momentum as the disk at the launch locations. Given the limited data against which these models can be tested, the simple-minded treatment of disk evolution is a virtue.

The inclusion of photoevaporation into disk models does not materially alter the derived observational constraints on the efficiency of disk angular momentum transport. Acceptable values of $\alpha$ derived by various authors range from $\alpha=2.5 \times 10^{-3}$ (Owen, Ercolano \& Clarke 2010) to $\alpha=10^{-2}$ (Gorti, Dullemond \& Hollenbach 2009), no different from earlier studies that ignored mass loss (Hartmann et al. 1998, Hueso \& Guillot 2005). Where photoevaporation matters - and can potentially be tested - is as an agent for disk dispersal. Clarke, Gendrin \& Sotomayor (2001) found that even low levels of mass loss, such as would be expected in an EUV-only photoevaporation scenario, would nonetheless have a dramatic effect on the disk, provided that the mass loss was concentrated at relatively small radii (as indeed occurs for EUV photoevaporation; Figure 9). In that case the disk evolves viscously until the accretion rate $\dot{M} \sim \dot{M}_{\text {wind }}$, at which point the wind opens up a gap in the disk near $r_{g}$. Subsequently the inner disk, now deprived of resupply from large radii, drains viscously onto the star, followed by a rapid dispersal of the outer disk by direct irradiation of the inner rim (Alexander, Clarke \& Pringle 2006b). The resulting "two time scale" behavior a slow period of evolution on the viscous time of the outer disk, followed by rapid dispersal on a time scale set by the inner disk - is in general accord with observations of prompt disk clearing (Wolk \& Walter 1996). Qualitatively similar evolution can be seen in more recent calculations that include X-ray and FUV contributions (Gorti, Dullemond \& Hollenbach 2009; Owen et al. 2010), although the generally higher mass loss rates imply a shift of the start of the dispersal phase to higher accretion rates and larger disk masses.

The most provocative finding of the studies of Gorti \& Hollenbach (2009) and Owen et al. (2010) is unquestionably the large disk-integrated mass loss rates. Since much of the mass loss is inferred to occur from large disk radii $(r \gtrsim 50 \mathrm{AU})$, an immediate implication is that the structure of isolated disks on these scales may often be determined largely by mass loss rather than by angular momentum transport (similar to the case of externally irradiated systems; Adams et al. 2004). There are also interesting predicted couplings between the mass loss rate and other observable properties of the system. Much of the FUV flux seen by the outer disk arises from the accretion of inner disk material onto the star, so in FUV-dominated photoevaporation there is an indirect coupling between the inner and outer disk (Matsuyama, Johnstone \& Hartmann 2003). If, instead, it is X-rays that dominate photoevaporative mass loss, then the linear dependence of $\dot{M}_{\text {wind }}$ on $L_{X}$ (Owen, Ercolano \& Clarke 2010) hints that the stellar X-ray luminosity function could be the 
fundamental determinant of disk evolution around low-mass stars (rather than, say, dispersion in the initial disk mass or angular momentum; Armitage, Clarke \& Palla 2003). In this view, the observed anti-correlation between accretion and stellar X-ray luminosity (Neuhaeuser et al. 1995) would derive from the suppression of accretion, and reduction in disk lifetime, for X-ray bright stars that drive particularly powerful winds (Drake et al. 2009; Owen, Ercolano \& Clarke 2010). Although this is a plausible coupling (and to some degree inescapable, if X-ray flux determines $M_{\text {wind }}$ to the extent implied by the results of Owen et al. 2010), the modeling is still at an early stage. Examples of possibly confounding effects include time evolution of the stellar X-ray spectrum, which may be affected by disk evolution via the influence of the disk on stellar rotation (Matt et al. 2010), and variations in wind loss rates due to dust settling and growth.

\section{INSTABILITIES AND OUTBURSTS}

There is both direct and statistical evidence that disk accretion onto young stars is episodic, particularly during the Class 1 or "protostellar" phase. The direct evidence comes from observations of outbursts, lasting from years to decades, in FU Orionis variables (Hartmann \& Kenyon 1996). The peak accretion rates during FU Orionis events are high enough - of the order of $M \sim 10^{-4} M_{\odot} \mathrm{yr}^{-1}$ - that if a typical star were to suffer a handful of such events, the total mass accreted would be comparable to estimated disk masses. That this might be the case is supported by statistical arguments, which show that the typical bolometric luminosity of protostars is smaller than would be expected from the infall rates and duration of the protostellar phase (Kenyon et al. 1990). This "luminosity problem" for protostars could be solved if the typical star accretes around $50 \%$ of its mass during less than $10 \%$ of its lifetime (Evans et al. 2009). In this Section we will review candidate mechanisms for generating variability, where by "variability" we really mean large-scale episodes of enhanced accretion violent enough to explain FU Orionis events and the luminosity problem. CTTs also exhibit a wealth of lower amplitude variability, likely attributable to the combined effects of fluctuations in $\dot{M}$ and to variable obscuration, which if it could be characterized well-enough (via long duration, uninterrupted monitoring) might ultimately be compared against simulations of turbulent accretion.

Models for FU Orionis outbursts generally invoke some combination of the following effects,

1. The rapid change in opacity near $T \sim 10^{4} \mathrm{~K}$, associated with the ionization of hydrogen.

2. A large change in MRI angular momentum transport efficiency at $T \sim 10^{3} \mathrm{~K}$, associated with the collisional ionization of alkali metals (3.2).

3. Gravitational instability, leading either to clump formation in the outer disk (followed by radial migration) or to heating in the inner disk.

That aspects of this physics are involved in FU Orionis is strongly indicated by observations; the inferred inner disk temperatures certainly exceed $10^{3} \mathrm{~K}$ or even $10^{4} \mathrm{~K}$, and protostellar disks in general are plausibly massive enough for self-gravity to be in play (Eisner et al. 2005).

Thermal instabilities have been thoroughly studied as a potential mechanism for FU Orionis outbursts (Bell \& Lin 1994). In equilibrium, the local heating rate $Q_{+}$of a viscous disk must balance the local cooling rate $Q_{-}$. If that equilibrium is unstable to small perturbations in the central temperature $T_{c}$, i.e. if,

$$
\frac{\mathrm{d} \log Q_{+}}{\mathrm{d} \log T_{c}}>\frac{\mathrm{d} \log Q_{-}}{\mathrm{d} \log T_{c}},
$$

then the disk is described as being thermally unstable (Pringle 1976). These conditions can be met for disks whose equilibrium mid-plane temperature is $T_{c} \sim 10^{4} \mathrm{~K}$, since around this temperature the ionization of hydrogen leads to very rapid changes in the opacity. If a disk possesses regions which are locally thermally unstable, it is possible (though not guaranteed) that it will also be 


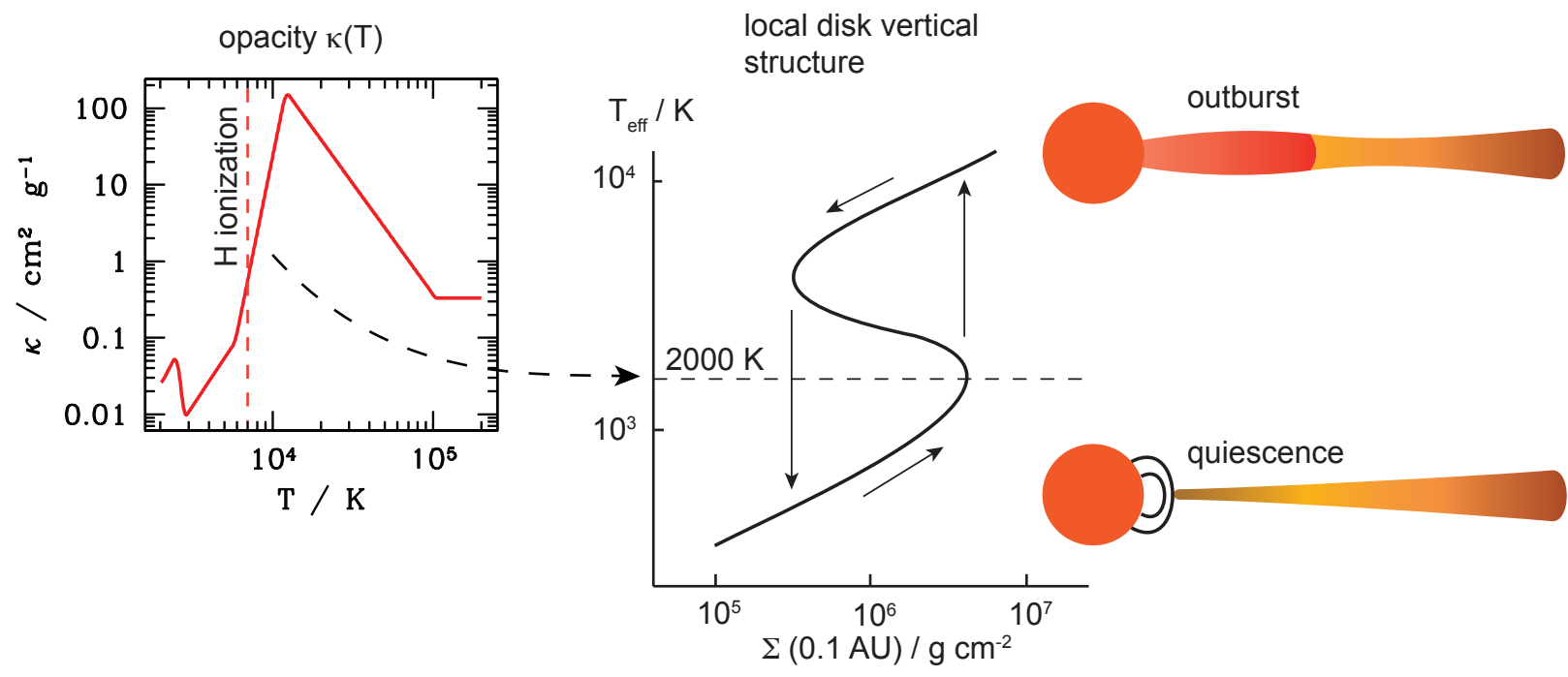

Figure 10: A classical disk thermal instability is a possibility if the accretion rate through the inner disk is such that that the mid-plane temperature approaches $T \sim 10^{4} \mathrm{~K}$, at which point hydrogen becomes ionized and the opacity $\kappa(T)$ rises rapidly. Under these conditions there can be multiple solutions (an "S-curve") for the local equilibrium vertical structure at fixed $r$ and $\Sigma$, with the stable possibilities being a quiescent state of low accretion rate, and an outburst state with a much higher temperature and accretion rate. Provided that these states are sufficiently well-separated, it is then possible to set up a global limit cycle in which the inner disk as a whole cycles between quiescent and outburst behavior.

unstable to the development of a globally organized limit cycle. The basic idea is illustrated in Figure 10. The disk cycles between a quiescent state, in which the mid-plane is primarily cool and neutral and the accretion rate is low, and an outburst state in which the disk is hot and rapidly accreting.

The existence of disk thermal instability is scarcely in doubt; the physics is simple and models based upon it are used successfully to explain dwarf nova outbursts (e.g. Hameury et al. 1998). However, the simplest models, in which thermal instability is the sole mechanism responsible for outbursts, only work if angular momentum transport in the inner disk is surprisingly inefficient. The one-dimensional models of Bell \& Lin (1994), which remain the most detailed attempt to model FU Orionis events, can match the observed time scales of outbursts if $\alpha \approx 10^{-4}$ when the mid-plane is neutral, and $\alpha \approx 10^{-3}$ when it is ionized. The low value of $\alpha$ in the outburst state, in particular, is hard to reconcile with either theoretical expectations for the efficiency of the MRI, or with observational constraints derived from protoplanetary disk lifetimes. As a result, and notwithstanding the otherwise satisfactory agreement between thermal instability models and observations (Bell et al. 1995), it appears more likely than not that additional physical processes contribute to the FU Orionis phenomenon. Two classes of ideas have been proposed. The first maintains the assumption that thermal instability is responsible for the phenomenology of outbursts, but drops the requirement that the inner disk be spontaneously unstable due to the slow axisymmetric accumulation of matter. Instead, it is proposed that some other process is able to dump matter rapidly into the inner disk, thereby triggering an outburst (Clarke, Lin \& Pringle 1990). Potential triggers include perturbations from companions (Bonnell \& Bastien 1992, Forgan \& Rice 2010, Pfalzner 
2008), or the inspiral of clumps formed in an outer self-gravitating region of the disk (Boley et al. 2010, Nayakshin 2010, Vorobyov \& Basu 2005, 2010).

External perturbations to the inner disk almost certainly do occur, but it is not known whether they are strong and frequent enough to account for FU Orionis events. An alternate class of models retains the "self-regulated" character of the classical thermal instability (Bell \& Lin 1994), and solves the time scale problem by associating the instability with MRI physics (and specifically the expected change in transport properties at $T_{c} \sim 10^{3} \mathrm{~K}$ ) at larger radii, where the viscous time scale is longer. The basic idea is to assume that a dead zone exists at $r \sim 1 \mathrm{AU}$, and that the strength of any residual transport in the mid-plane layer is low (this is an important assumption, since a significant residual viscosity would allow the disk to reach a steady-state; Terquem 2008). Such a dead zone is massive and potentially unstable, since if it could once be heated above $T_{c} \sim 10^{3} \mathrm{~K}$ the onset of the MRI would subsequently be able to maintain a high dissipation state until much of the mass in the inner disk had been accreted. Self-gravity in the dead zone, caused by the slow accumulation of mass there, could provide the heating source necessary to realize the latent instability (Armitage, Livio \& Pringle 2001; Gammie 1999).

These early ideas of how a dead zone might give rise to outbursts have recently been studied in detail, using both two-dimensional hydrodynamic simulations (Zhu et al. 2009) and one dimensional time-dependent disk models (Zhu, Hartmann \& Gammie 2010; Zhu et al. 2010). Zhu et al. (2010) find that protostellar accretion is liable to FU Orionis type events for infall rates of the order of $\dot{M}_{\text {infall }} \sim 10^{-6} M_{\odot} \mathrm{yr}^{-1}$, with dead zone instabilities being triggered at radii between $1 \mathrm{AU}$ and 10 AU. As noted above the mechanism is closely related to the classical disk thermal instability, with the main distinction being that the bistable structure of the disk derives from a rapid change in $\alpha$ with $T_{c}$ due to MRI physics, rather than from a rapid change in $\kappa$ as hydrogen is ionized. The models appear to be consistent with the main observed properties of FU Orionis events, with the most distinctive prediction being the survival of a relatively massive belt of gas in the dead zone region after outbursts have ceased (Armitage, Livio \& Pringle 2001; Zhu, Hartmann \& Gammie 2010). This may be observable with high angular resolution observations with the EVLA or ALMA (Zhu, Hartmann \& Gammie 2010).

\section{SUMMARY AND OUTLOOK}

On a optimistic reading, work to date has succeeded in identifying the basic physical processes responsible for protoplanetary disk evolution. The important angular momentum processes are the magnetorotational instability and disk self-gravity, with hydrodynamic instabilities associated with radial entropy gradients also possibly contributing. None of these processes behaves like an $\alpha$ viscosity, but we have at least an outline of how their angular momentum transport efficiencies scale with disk conditions. The ionization state (for the MRI), and the local cooling time scale (for self-gravity and baroclinic instabilities) are critical. Photoevaporation is almost certainly able to disperse disks, and may rival angular momentum transport for primacy in determining disk evolution, although the relative importance of FUV, EUV and X-ray photons remains subject to debate. Simplified models that combine these processes are broadly consistent with observations of disk populations (lifetimes, accretion rates and so on), and may be able to account for the eruptive behavior that appears to be common during the protostellar accretion phase.

There are abundant reasons to suspect that this accounting of important physics is incomplete. Most obviously, for want of space, we have restricted ourselves to considering viscous disk models, and have ignored the alternate possibility that magnetic winds or magnetic braking are responsible for significant disk evolution. Even if the above sketch is basically correct, however, there is much to be done. None of the important angular momentum transport processes is understood at a level adequate to securely predict properties of disks - such as the amplitude of turbulent velocities or the depth of gaps carved by planets - that may be observable in the near future. Of the many open 
theoretical questions, three stand out as being particularly pressing,

1. What is the non-linear outcome of the MRI across the full range of non-ideal conditions prevalent in protoplanetary disks? There has been a dramatic recent shift in our understanding of how the MRI behaves in (near)-ideal MHD, and there is a need to revisit the influence of Ohmic and ambipolar diffusion, and the Hall effect, in light of those results and new numerical techniques. The importance of global effects, over long time scales, remains poorly known.

2. How does the inclusion of magnetic fields affect the fragmentation of self-gravitating disks, and the efficiency of angular momentum transport in the regime where fragmentation is avoided? Preliminary work (Fromang et al. 2004) suggests that significant changes to hydrodynamic results are likely when magnetic fields are included, which could be important for the dynamics of disks and the formation of sub-stellar objects at large radii.

3. How do turbulent disks interact with planets? Many processes known to be of fundamental importance for planet formation - including migration, gap formation, and resonant capture - involve disks, and their detailed study probably requires explicit specification of the nature of disk turbulence.

The last of these is emblematic of a more general point; in protoplanetary disks the strength and nature of turbulence is of central importance quite independent of its role (if any) in disk dynamics. The effects of MHD or hydrodynamic turbulence on mixing, on the formation or dynamics of planetesimals, or even on the thermal processing of solids, are of at least equal interest for planet formation.

The theoretical and computational prerequisites for tackling problems such as those listed above are already in place, and rapid progress is possible. Alas, it requires not merely optimism, but irrational exuberance, to believe that even the successful execution of such a theoretical program will bring us close to a confident understanding of how disks evolve. Everything we know about disks suggests that no single, simple process dominates their dynamics. Rather, multiple transport and mass loss processes - each complex in its own right, and coupled to other problems in star formation and disk physics - contribute at different times and radii. Divining how disks evolve, and hence what can reasonably be inferred as to the initial conditions for planet formation, will thus require a much closer interplay of observations and theory than has been achieved to date. Qualitative advances in understanding could be triggered by new observations in any of several areas, including,

1. Direct measurements of, or stringent limits on, turbulent velocity fields within disks. Constraints on the strength and structure of magnetic fields would be just as valuable.

2. Detection of non-axisymmetric structure. This is only expected at large radii, in disks that are massive and self-gravitating, though more speculative possibilities such as significant disk eccentricity cannot be ruled out.

3. Statistical studies of disks that are based on gas, rather than dust, tracers.

4. A direct measure of the wind mass loss rate, and velocity structure, for a sample of disks undergoing photoevaporation driven by the central star.

5. Any constraint on the structure of disks at the most uncertain, and important (for planet formation), scale, between $1 \mathrm{AU}$ and $10 \mathrm{AU}$. This region is where a dead zone may (or may not) exist, and where the roadblock to ab initio theory, posed by the coupling of MHD processes to dust physics, currently appears most intractable.

Encouragingly, limited observational results are already available for most of this wish-list, along with enticing projections of what $A L M A$ and other future facilities ought to be able to achieve (e.g. Cossins, Lodato \& Testi 2010; Wolf \& D'Angelo 2005). The resolution and sensitivity of ALMA will 
also surely uncover entirely unexpected features of protoplanetary disks, that we hope will point the way toward a better understanding of the dynamics of these objects.

\section{ACKNOWLEDGMENTS}

I thank my colleagues at the Isaac Newton Institute for Mathematical Sciences at Cambridge University, the Racah Institute of Physics at the Hebrew University, the Kavli Institute for Theoretical Physics at the University of Santa Barbara, and the Kavli Institute of Astronomy and Astrophysics at Peking University, for hospitality and advice on many of the topics discussed here. Elena Rossi, Richard Alexander, Kris Beckwith, Aaron Boley, Kees Dullemond, Uma Gorti, Geoffroy Lesur, Giuseppe Lodato, Gordon Ogilvie, Ken Rice and Zhoahuan Zhu all provided invaluable assistance toward the completion of the review. My work has been supported, in part, by the National Science Foundation, by NASA, and by the University of Colorado's Council on Research and Creative Work.

\section{References}

1. Adams FC, Hollenbach D, Laughlin G, Gorti U 2004. Ap.J. 611:360

2. Afshordi N, Mukhopadhyay B, Narayan R 2005. Ap.J. 629:373

3. Alexander RD 2008. MNRAS 391:L64

4. Alexander RD, Armitage PJ 2009. Ap.J. 704:989

5. Alexander RD, Clarke CJ, Pringle JE 2005. MNRAS 358:283

6. Alexander RD, Clarke CJ, Pringle JE 2006. MNRAS 369:216

7. Alexander RD, Clarke CJ, Pringle JE 2006. MNRAS 369:229

8. Andrews SM, Wilner DJ, Hughes AM, Qi C, Dullemond CP 2009. Ap.J. 700:1502

9. Armitage PJ 1998. Ap.J 501:L189

10. Armitage PJ 2000. A\&A 362:968

11. Armitage PJ 2010. Astrophysics of Planet Formation, Cambridge, UK: Cambridge University Press. $294 \mathrm{pp}$.

12. Armitage PJ, Clarke CJ, Palla F 2003. MNRAS 342:1139

13. Armitage PJ, Livio M, Pringle JE 2001. MNRAS 324:705

14. Bai XN, Goodman J 2009. Ap.J. 701:737

15. Balbus SA 2010. Physical Processes in Circumstellar Disks around Young Stars, ed. PJV Garcia (Chicago, University of Chicago Press)

16. Balbus SA, Hawley JF 1991. Ap.J. 376:214

17. Balbus SA, Hawley JF 1998. Rev. Mod. Phys. 70:1

18. Balbus SA, Papaloizou JCB 1999. Ap.J. 521:650

19. Balbus SA, Terquem C 2001. Ap.J. 552:235

20. Bally J, Scoville NZ 1982. Ap.J. 255:497

21. Barge P, Sommeria J 1995. A\&A 295:L1

22. Barranco JA, Marcus PS 2005. Ap.J. 623:1157

23. Beckwith K, Armitage PJ, Simon JB 2011. MNRAS in press

24. Begelman MC, McKee CF, Shields GA 1983. Ap.J. 271:70 
25. Bell KR, Cassen PM, Klahr HH, Henning Th 1997. Ap.J 486:372

26. Bell KR, Lin DNC 1994. Ap.J. 427:987

27. Bell KR, Lin DNC, Hartmann LW, Kenyon SJ 1995. Ap.J. 444:376

28. Blaes OM, Balbus SA 1994. Ap.J. 421:163

29. Boley AC 2009. Ap.J. 695:L53

30. Boley AC, Durisen RH 2008. Ap.J. 685:1193

31. Boley AC, Hayfield T, Mayer L, Durisen RH 2010. Icarus, 207:509

32. Boley AC, Mejía AC, Durisen RH, Cai K, Pickett MK, D’Alessio P 2006. Ap.J. 651:517

33. Bonnell I, Bastien P 1992. Ap.J. 401:L31

34. Boss AP 1997. Science 276:1836

35. Boss AP 2008. Ap.J. 677:607

36. Boss AP, Durisen RH 2005. Ap.J. 621:L137

37. Brandenburg A, Nordlund A, Stein RF, Torkelsson U 1995. Ap.J. 446:741

38. Brandenburg A, Zweibel EG 1994. Ap.J. 427:L91

39. Cai K, Durisen RH, Boley AC, Pickett MK, Mejía AC 2008. Ap.J. 673:1138

40. Cai K, Durisen RH, Michael S, Boley AC, Mejía AC, Pickett MK, D’Alessio P 2006. Ap.J 636:L149

41. Cai K, Pickett MK, Durisen RH, Milne AM 2010. Ap.J. 716:L176

42. Calvet N, D'Alessio P, Hartmann L, Wilner D, Walsh A, Sitko M 2002. Ap.J. 568:1008

43. Calvet N, Gullbring E 1998. Ap.J 509:802

44. Carballido A, Stone JM, Pringle JE 2005. MNRAS 358:1055

45. Chagelishvili GD, Zahn JP, Tevzadze AG, Lominadze JG 2003. A\&A 402:401

46. Chavanis PH 2000. A\&̈A 356:1089

47. Chiang EI, Goldreich P 1997. Ap.J. 490:368

48. Chiang E, Youdin AN 2010. Annu. Rev. Earth Plan. Sci. 38:493

49. Clarke CJ 2007. MNRAS 376:1350

50. Clarke CJ 2009. MNRAS 396:1066

51. Clarke CJ, Gendrin A, Sotomayor M 2001. MNRAS 328:485

52. Clarke CJ, Lin DNC, Pringle JE 1990. MNRAS 242:439

53. Clarke CJ, Pringle JE 1988. MNRAS 235:365

54. Cossins P, Lodato G, Clake CJ 2009. MNRAS 393:1157

55. Cossins P, Lodato G, Clake CJ 2010. MNRAS 401:2587

56. Cossins P, Lodato G, Testi L 2010. MNRAS 407:181

57. Cuzzi JN, Dobrovolskis AR, Champney JM 1993. Icarus 106:102

58. D'Alessio P, Calvet N, Hartmann L 2001. Ap.J 553:321

59. D'Alessio P, Canto J., Calvet N, Lizano S 1998. Ap.J 500:411

60. Davis SW, Stone JM, Pessah ME 2010. Ap.J. 713:52

61. Desch SJ 2004. Ap.J. 608:509

62. Draine BT, Sutin B 1987. Ap.J 320:803

63. Drake JJ, Ercolano B, Flaccomio E, Micela G 2009. Ap.J. 699:L35 
64. Dubrulle B, Morfill G, Sterzik M 1995. Icarus 114:237

65. Dullemond CP, Dominik C 2005. A\&A 434:971

66. Dzyurkevich N, Flock M, Turner NJ, Klahr H, Henning T 2010. AछA 515:70

67. Eisner JA, Hillenbrand LA, Carpenter JM, Wolf S 2005. Ap.J. 635:396

68. Ercolano B, Drake JJ, Raymond JC, Clarke C 2008. Ap.J. 688:398

69. Ercolano B, Clarke CJ, Drake JJ 2009. Ap.J. 699:1639

70. Ercolano B, Owen JE 2010. MNRAS 406:1553

71. Evans NJ, et al. 2009. Ap.J.S. 181:321

72. Fatuzzo M, Adams FC 2008. Ap.J. 675:1361

73. Fleming TP, Stone JM, Hawley JF 2000. Ap.J. 530:464

74. Fleming T, Stone JM 2003. Ap.J. 585:908

75. Font AS, McCarthy IG, Johnstone D, Ballantyne DR 2004. Ap.J. 607:890

76. Forgan D, Rice K 2010. MNRAS 402:1349

77. Forgan D, Rice K, Cossins P, Lodato G 2010. MNRAS in press

78. Frank J, King A, Raine DJ 2002. Accretion Power in Astrophysics, Cambridge, UK: Cambridge University Press. 398 pp. 3rd ed.

79. Fromang S, Balbus SA, Terquem C, De Villiers JP 2004. Ap.J. 616:364

82. Fromang S, Nelson RP 2006. A\&A 457:343

81. Fromang S, Nelson RP 2009. A\&A 496:597

82. Fromang S, Papaloizou J 2006. A\&A 452:751

83. Fromang S, Papaloizou J 2007. A $\mathscr{G} A$ 476:1113

84. Fromang S, Papaloizou J, Lesur G, Heinemann T 2007. A\&A 476:1123

85. Fromang S, Terquem C, Balbus SA 2002. MNRAS 329:18

86. Gammie CF 1996. Ap.J. 457:355

87. Gammie CF 1999. Astrophysical Discs, ed JA Sellwood, J Goodman (Astronomical Society of the Pacific), p. 122

88. Gammie CF 2001. Ap.J. 553:174

89. Geers VC, et al. 2006. $A \& A$ 459:545

90. Glassgold AE, Najita J, Igea J 1997. Ap.J. 480:344

91. Goldreich P, Tremaine S 1980. Ap.J. 241:425

92. Goodman J, Rafikov RR 2001. Ap.J. 552:793

93. Gorti U, Hollenbach D 2008. Ap.J. 683:287

94. Gorti U, Hollenbach D 2009. Ap.J. 690:1539

95. Gorti U, Dullemond CP, Hollenbach D 2009. Ap.J. 705:1237

96. Guan X, Gammie CF, Simon JB, Johnson BM 2009. Ap.J. 694:1010

97. Güdel M, et al. 2007. A\& A 468:353

98. Haisch KE Jr., Lada EA, Lada CJ 2001. Ap. J. Lett. 553:153

99. Hameury JM, Menou K, Dubus G, Lasota JP, Hure JM 1998. MNRAS 298:1048

100. Harsono D, Alexander RD, Levin Y 2010. MNRAS in press 
101. Hartmann L, Calvet N, Gullbring E, D’Alessio P 1998. Ap. J. 495:385

102. Hartmann L, Kenyon SJ 1996. Annu. Rev. Astron. Astrophys. 34:207

103. Hawley JF, Gammie CF, Balbus SA 1995. Ap.J. 440:742

104. Hawley JF, Stone JM 1998. Ap.J. 501:758

105. Hayashi C 1981. Prog. Theor. Phys. Suppl. 70:35

106. Henney WJ, O’Dell CR, Meaburn J, Garrington ST, Lopez JA 2002. Ap.J. 566:315

107. Herczeg GJ, Najita JR, Hillenbrand LA, Pascucci I 2008. Ap.J. 670:509

108. Hollenbach D, Johnstone D, Lizano S, Shu F 1994. Ap.J. 428:654

109. Hollenbach D, Johnstone D, Shu F 1993. Massive Stars: Their Lives in the Interstellar Medium. eds JP Cassinelli, EB Churchwell, (ASP Conference Series) 35, p. 26

110. Hubeny I 1990. Ap.J. 351:632

111. Hueso R, Guillot T 2005. A\&A 442:703

112. Hughes AM, Wilner DJ, Andrews SM, Qi C, Hogerheijde MR 2010. Ap.J. in press

113. Hughes AM, Wilner DJ, Cho J, Marrone DP, Lazarian A, Andrews SM, Rao R 2009. Ap.J. 704:1204

114. Ioannou PJ, Kakouris A 2001. Ap.J. 550:931

115. Igea J, Glassgold AE 1999. Ap.J. 518:848

116. Ilgner M, Nelson RP 2006. A\& A 445:205

117. Ilgner M, Nelson RP 2008. A $\& A$ 483:815

118. Inutsuka S, Sano T 2005. Ap.J. 628:L155

119. Ji H, Burin M, Schartman E, Goodman J 2006. Nature 444:343

120. Jin L 1996. Ap.J. 457:798

121. Johansen A, Klahr H 2005. Ap.J. 634:1353

122. Johansen A, Klahr H, Mee AJ 2006. MNRAS 370:L71

123. Johansen A, Levin Y 2008. Ap.J. 490:501

124. Johansen A, Youdin A, Mac Low M-M 2009. Ap.J. 704:L75

125. Johnson BM, Gammie CF 2003. Ap.J. 597:131

126. Johnson BM, Gammie CF 2005. Ap.J. 635:149

127. Johnstone D, Hollenbach D, Bally J 1998. Ap.J. 499:758

128. Joung MKR, Mac Low MM, Ebel DS 2004. Ap.J. 606:532

129. Kamp I, Sammar F 2004. A $\& A$ 427:561

130. Kenyon SJ, Hartmann LW, Strom KM, Strom SE 1990. AJ 99:869

131. Klahr HH, Bodenheimer P 2003. Ap.J. 582:869

132. King AR, Pringle JE 2010. MNRAS 404:1903

133. Königl A 1991. Ap.J. 370:L39

134. Königl A, Salmeron R 2010. Physical Processes in Circumstellar Disks around Young Stars, ed. PJV Garcia (Chicago, University of Chicago Press)

135. Kratter KM, Matzner CD, Krumholz MR, Klein, RI 2010. Ap.J. 708:1585

136. Kratter KM, Murray-Clay RA, Youdin AN 2010. Ap.J. 710:1375

137. Krumholz MR, Klein RI, McKee CF 2007. Ap.J. 656:959 
138. Kunz MW, Balbus SA 2004. MNRAS 348:355

139. Latter HN, Bonart JF, Balbus SA 2010. MNRAS 405:1831

140. Laughlin G, Bodenheimer P 1994. Ap.J. 436: 335

141. Lesur G, Longaretti PY 2005. Ap.J. 444:25

142. Lesur G, Ogilvie GI 2010. MNRAS 404:L64

143. Lesur G, Papaloizou JCB 2010. A $\& A$ 513:60

144. Levin Y 2007. MNRAS 374:515

145. Liffman K 2003. Pub. Astron. Soc. Aus. 20:337

146. Lin DNC, Papaloizou J 1980. MNRAS 191:37

147. Lin DNC, Pringle JE 1987. MNRAS 225:607

148. Lodato G, Rice WKM 2004. MNRAS 351:630

149. Lodato G, Rice WKM 2005. MNRAS 358:1489

150. Longaretti PY, Lesur G 2010. A\&A 516:A51

151. Lubow SH, D'Angelo G 2006. Ap.J. 641:526

152. Lubow SH, Ida S 2010. Exoplanets, ed. S Seager, University of Arizona Press

153. Lynden-Bell D, Kalnajs AJ 1962. MNRAS 157:1

154. Lynden-Bell D, Pringle JE 1974. MNRAS 168:603

155. Mac Low MM, Norman ML, Konigl A, Wardle M 1995. Ap.J. 442:726

156. Mathis JS, Rumpl W, Nordsieck KH 1977. Ap.J. 217:425

157. Matsuyama I, Johnstone D, Hartmann L 2003. Ap.J. 582:893

158. Matt SP, Pinzón G, de la Reza R, Greene TP 2010. Ap.J. 714:989

159. Matzner CD, Levin Y 2005. Ap.J. 628: 817

160. Mayer L, Lufkin G, Quinn T, Wadsley J 2007. Ap.J. 661:L77

161. Meru F, Bate MR 2010. MNRAS in press

162. Mejía AC, Durisen RH, Pickett MK, Cai K 2005. Ap.J. 619:1098

163. Morbidelli A, Crida A 2007. Icarus 191:158

164. Movshovitz N, Bodenheimer P, Podolak M, Lissauer JJ 2010. Icarus 209:616

165. Nayakshin S 2010. MNRAS 408:L36

166. Nelson AF, Benz W, Ruzmaikina TV 2000. Ap.J. 529:357

167. Neuhaeuser R, Sterzik MF, Schmitt JHMM, Wichmann R, Krautter J 1995. A\&A 297:391

168. O’Dell CR, Wen Z, Hu X 1993. Ap.J. 410:696

169. Oishi JS, Mac Low MM 2009. Ap.J. 704:1239

170. Oliveira I, et al. 2010. Ap.J. 714:778

171. Oppenheimer M, Dalgarno A 1974. Ap.J. 192:29

172. Owen JE, Ercolano B, Clarke CJ 2010. $M N R A S$ in press

173. Owen JE, Ercolano B, Clarke CJ, Alexander RD 2010. MNRAS 401:1415

174. Paczyński B 1978. Acta Astronomica 28:91

175. Papaloizou JC, Savonije GJ 1991. MNRAS 248:353

176. Pascucci I, Sterzik M 2009. Ap.J. 702:724 
177. Perez-Becker D, Chiang E 2010. Ap.J. in press

178. Pessah ME, Chan C, Psaltis D 2007. Ap.J 668:L51

179. Petersen MR, Stewart GR, Julien K 2007. Ap.J. 658:1252

180. Petersen MR, Julien K, Stewart GR 2007. Ap.J. 658:1236

181. Pfalzner S 2008. A\& A 492:735

182. Pickett BK, Cassen P, Durisen RH, Link, R 1998. Ap.J. 504:468

183. Pickett BK, Cassen P, Durisen RH, Link, R 2000. Ap.J. 529:1034

184. Preibisch T, et al. 2005. Ap.J.S. 160:401

185. Pringle JE 1976. MNRAS 177:65

186. Pringle JE 1981. Annu. Rev. Astron. Astrophys. 19:137

187. Rafikov R 2005. Ap.J. 621:L69

188. Rafikov R 2009. Ap.J. 704:281

189. Rebusco P, Umurhan OM, Kluźniak W, Regev O 2009. Physics of Fluids 21:076601

190. Rice WKM, Armitage PJ 2009. MNRAS 396:228

191. Rice WKM, Armitage PJ, Bate MR, Bonnell IA 2003. MNRAS 339:1025

192. Rice WKM, Wood K, Armitage PJ, Whitney BA, Bjorkman JE 2003. MNRAS 342:79

193. Rice WKM, Lodato G, Armitage PJ 2005. MNRAS 364:L56

194. Rice WKM, Mayo JH, Armitage PJ 2010. MNRAS 402:1740

195. Richling S, Yorke HW 1997. Ap.J. 327:317

196. Richling S, Yorke HW 2000. Ap.J. 539:258

197. Röllig M et al. 2007. A\& A 467:187

198. Salmeron R, Wardle M 2005. MNRAS 361:45

199. Salmeron R, Wardle M 2008. MNRAS 388:1223

200. Sano T, Inutsuka S 2001. Ap.J. 561:L179

201. Sano T, Miyama SM 1999. Ap.J. 515:776

202. Sano T, Miyama SM, Umebayashi T, Nakano T 2000. Ap.J. 543:486

203. Sano T, Stone JM 2002. Ap.J. 577:534

204. Sari R, Goldreich P 2004. Ap.J. 606:L77

205. Scott ERD 2007. AREPS 35:577

206. Semenov D, Henning Th, Helling Ch, Ilgner M, Sedlmayr E 2003. A\&A 410:611

207. Semenov D, Wiebe D, Henning T 2004. A $\& A$ 417:93

208. Shakura NI, Sunyaev RA 1973. A\&A 24:337

209. Shi J, Krolik JH, Hirose S 2010. Ap.J. 708:1716

210. Shlosman I, Begelman MC 1987. Nature 329:810

211. Shu FH, Johnstone D, Hollenbach D 1993. Icarus 106:92

212. Simon JB, Hawley JF 2009. Ap.J. 707:833

213. Simon JB, Hawley JF, Beckwith K 2009. Ap.J. 690:974

214. Simon JB, Hawley JF, Beckwith K 2010. Ap.J. submitted

215. Sorathia KA, Reynolds CS, Armitage PJ 2010. Ap.J. 712:1241 
216. Spitzer L 1962. Physics of Fully Ionized Gases. New York: Interscience, 2nd ed.

217. Spitzer L, Tomasko MG 1968. Ap.J. 152: 971

218. Stamatellos D, Hubber DA, Whitworth A 2007. MNRAS 382:L30

219. Stamatellos D, Whitworth AP 2008. A $\& A$ 480:879

220. Stamatellos D, Whitworth AP 2009. MNRAS 392:413

221. Stepinski TF 1992. Icarus 97:130

222. Stone JM, Hawley JF, Gammie CF, Balbus SA 1996. Ap.J. 463:656

223. Störzer H, Hollenbach D 1999. Ap.J. 515:669

224. Suzuki TK, Inutsuka S 2009. Ap.J. 691:L49

225. Tanga P, Babiano A, Dubrulle B, Provenzale A 1996. Icarus 121:158

226. Terquem CEJMLJ 2008. MNRAS 689:532

227. Toomre A 1964. Ap.J. 139:1217

228. Tout CA, Pringle JE 1996. MNRAS 281:219

229. Turner NJ, Carballido A, Sano T 2010. Ap.J. 708:188

230. Turner NJ, Drake JF 2009. Ap.J. 703:2152

231. Turner NJ, Sano T 2008. Ap.J. 679:L131

232. Turner NJ, Sano T, Dziourkevitch N 2007. Ap.J. 659:729

233. Turner NJ, Willacy K, Bryden G, Yorke HW 2006. Ap.J. 639:1218

234. Umebayashi T, Nakano T 1981. PASJ 33:617

235. Umebayashi T, Nakano T 2009. Ap.J. 690:69

236. Uzdensky DA, Goodman J 2008. Ap.J. 682:608

237. Vasconcelos MJ, Cerqueira AH, Plana H, Raga AC, Morisset C 2005. AJ 130:1707

238. Vasyunin AI, Semenov D, Henning T. Wakelam V, Herbst E, Sobolev AM 2008. Ap.J. 672:629

239. Vorobyov EI, Basu S 2005. Ap.J. 633:L137

240. Vorobyov EI, Basu S 2006. Ap.J. 650:956

241. Vorobyov EI, Basu S 2010. Ap.J. 719:1896

242. Wardle M 1999. MNRAS 307:849

243. Williams JP, Cieza LA 2011. Annu. Rev. Astron. Astrophys. this volume

244. Weidenschilling SJ 1977. Astrophysics and Space Science 51:153

245. Woitke P, Kamp I, Thi WF 2009. A\&A 501:383

246. Wolf S, D'Angelo G 2005. Ap.J. 619:1114

247. Wolk SJ, Walter FM 1996. AJ 111:2066

248. Wyatt MC 2008. Annu. Rev. Astron. Astrophys. 46:339

249. Yorke HW 1993. Massive Stars: Their Lives in the Interstellar Medium. eds JP Cassinelli, EB Churchwell, (ASP Conference Series) 35, p. 45

250. Youdin AN, Lithwick Y 2007. Icarus 192:588

251. Zhu Z, Hartmann L, Gammie C, McKinney JC 2009. Ap.J. 701:620

252. Zhu Z, Hartmann L, Gammie C 2010. Ap.J. 713:1143

253. Zhu Z, Hartmann L, Gammie CF, Book LG, Simon JB, Engelhard E 2010. Ap.J. 713:1134 